\title{
The functional architecture of S1 during touch observation described with 7 T fMRI
}

\author{
Esther Kuehn • Karsten Mueller • Robert Turner • \\ Simone Schütz-Bosbach
}

Received: 7 September 2012/ Accepted: 20 November 2012/Published online: 3 January 2013

(C) The Author(s) 2012. This article is published with open access at Springerlink.com

\begin{abstract}
Recent studies indicate that the primary somatosensory cortex (S1) is active not only when touch is physically perceived but also when it is merely observed to be experienced by another person. This social responsivity of S1 has important implications for our understanding of S1 functioning. However, S1 activity during touch observation has not been characterized in great detail to date. We focused on two features of the S1 functional architecture during touch observation, namely the topographical arrangement of index and middle finger receptive fields (RFs), and their dynamic shrinkage during concurrent activation. Both features have important implications for human behavior. We conducted two fMRI studies at $7 \mathrm{~T}$, one where touch was physically perceived, and one where touch was observed. In the two experiments, participants either had their index finger and/or middle finger stimulated using paintbrushes, or just observed similar touch events on video. Our data show that observing and physically experiencing touch elicits overlapping activity changes in S1. In addition, observing touch to the index finger or the middle finger alone evoked topographically arranged activation foci in S1. Importantly, when co-activated, the index and middle finger RFs not only shrank during physical touch perception, but also during touch observation. Our data, therefore, indicate a similarity between the functional architecture of S1 during touch observation and physical touch perception with respect to single-digit
\end{abstract}

Electronic supplementary material The online version of this article (doi:10.1007/s00429-012-0489-z) contains supplementary material, which is available to authorized users.

E. Kuehn $(\bowtie) \cdot$ K. Mueller $\cdot$ R. Turner $\cdot$ S. Schütz-Bosbach Max Planck Institute for Human Cognitive and Brain Sciences, Stephanstr. 1a, 04103 Leipzig, Germany

e-mail: ekuehn@cbs.mpg.de topography and RF shrinkage. These results may allow the tentative conclusion that even primary somatosensory experiences, such as physical touch perception, can be shared amongst individuals.

Keywords Empathy · Primary somatosensory cortex · Mirror neuron system - Shared representations .

Suppressive interactions - Receptive field

\section{Introduction}

In recent years, the unisensory and private character of many primary sensory brain areas has increasingly been questioned. Traditionally, primary sensory brain areas, such as the primary somatosensory cortex (S1), have been assumed to be unisensory in character, and to only respond to modality-specific input; however, recent research has provided evidence for their multisensory response properties (Kayser 2010). The responsivity of primary sensory brain areas to multimodal input has even been called a "revolution in multisensory research" (Driver and Noesselt 2008), and S1 seems to be part of it. An increasing amount of evidence shows that $\mathrm{S} 1$ activity is not only influenced by direct somatosensory input, but is also modulated by other factors such as attention (Eimer et al. 2001; Hsiao et al. 1993; Macaluso et al. 2002), reward (Pleger et al. 2008), spatial processing (Eimer et al. 2001), or visual stimulation (Dionne et al. 2010; Zhou and Fuster 1997). As a consequence, the emerging view in cognitive neuroscience is that $\mathrm{S} 1$ can no longer be regarded as a strictly unisensory brain area, but rather as an area whose activity levels can be shaped by multiple environmental inputs.

Yet another quantum leap for our understanding of S1 is that recent studies have shown a specific influence of social 
cues on its functioning. Not only has evidence been provided that viewing the body compared with viewing an object can influence S1 processing during physical touch perception (Cardini et al. 2011; Fiorio and Haggard 2005; Longo et al. 2011), but it has also been shown that viewing touch to a body, without physically perceiving touch at all, can increase S1 activity levels (Blakemore et al. 2005; Ebisch et al. 2008; Kuehn et al. 2012; Schaefer et al. 2009, 2012). S1 activity changes during touch observation were shown to be stronger when human touch compared with object touch was observed (Blakemore et al. 2005), and a specifically social responsivity of $\mathrm{S} 1$ during touch observation has been assumed (Kuehn et al. 2012; Rossetti et al. 2012).

Importantly, active voxels in S1 that were triggered by touch observation were shown to overlap with voxels activated during physical touch perception (Blakemore et al. 2005; Ebisch et al. 2008; Schaefer et al. 2009). For example, using functional magnetic resonance imaging (fMRI), Blakemore and colleagues showed that somatotopically specific areas in S1 were activated when touch to another person's face and neck was observed and that such activity changes overlapped with those areas that were activated during the physical perception of touch to the subject's own neck and face (Blakemore et al. 2005). This is similar to the neuronal "resonance" responses reported for the motor system (Buccino et al. 2001; Gazzola and Keysers 2009; Mukamel et al. 2010), and the emotional system (CorradiDell'Acqua et al. 2011; Singer et al. 2004), where similar areas in the brain were shown to activate during action observation and execution, and emotion observation and perception, respectively. It is assumed that such resonance responses allow a basic understanding of the observed action or emotion, respectively (Bernhardt and Singer 2012; Fabbri-Destro and Rizzolatti 2008). According to this logic, S1 activity during touch observation should allow a basic understanding of another person's somatosensory experiences as has recently been proposed (Avenanti et al. 2007; Keysers et al. 2010).

An interesting resulting question is how activity changes in S1 during touch observation can be characterized. Which features of the functional architecture of S1 are shared between physically perceiving and observing touch, and which only occur in one or the other condition? Based on recent neuroimaging research on the human somatosensory system, two features of the functional architecture of S1 seem to be of specific importance in this respect. On the one hand, the topographical arrangement of cortical receptive fields (RFs) in $\mathrm{S} 1$ plays an important role in our understanding of somatosensory processing. S1 activity during physical touch perception represents the contralateral side of the human body in a mediolateral sequence (Blankenburg et al. 2003; Gardner EK 2008; Kaas et al.
2002; Krause et al. 2001), and has been shown to make an important contribution to the ability to localize tactile stimuli on the skin (Beauchamp et al. 2009; Chen et al. 2003, 2007; Schweizer et al. 2001). Changes in the topographical arrangement of single-digit RFs in S1 can, for example, lead to a diminished ability to assign touch to a particular digit (Braun et al. 2000; Schweizer et al. 2001).

On the other hand, suppressive interactions between coactivated RFs in S1 have often been used to describe the functional architecture of S1. RFs in S1 react within milliseconds to sensory inputs given to surrounding RFs. For instance, the RF of one digit contracts when other digits are stimulated simultaneously and rapidly expands again when the digit is stimulated alone. This shrinking and re-enlarging of S1 RFs, depending on the tactile input given to surrounding RFs, has been demonstrated in studies on cats, mice, and monkeys (Friedman et al. 2008; Moore et al. 1999; Zarzecki and Wiggin 1982), and is posited to reflect a wide-spread cortical mechanism, which results in increased perceived stimulus contrast (Dykes 1983; Falkner et al. 2010; Jones 1993; Moore et al. 1999). Suppressive interactions during physical touch perception have also been investigated in multiple human studies (Biermann et al. 1998; Braun et al. 2002; Gandevia et al. 1983; Haavik Taylor and Murphy 2007; Hoechstetter et al. 2001; Ishibashi et al. 2000; Naka et al. 1998; Ruben et al. 2006; Tanosaki et al. 2002; Torquati et al. 2003), where the arithmetic sum of $\mathrm{S} 1$ signals measured during separate single-digit stimulations of two fingers was compared with the signal change during the stimulation of both fingers at once. In these studies, decreased signal strength during double-digit stimulation, compared with the sum of signals from separate single-digit stimulations, indicated how much the RFs contracted. Also in humans, such RF shrinkages presumably mediated by suppressive interactions, are assumed to positively relate to perceived stimulus contrast (Braun et al. 2002; Cardini et al. 2011; Puts et al. 2011).

Given that the aim of the present study was to characterize the functional architecture of S1 during touch observation along these two dimensions (topographical arrangement of RFs and suppressive interactions between RFs), the use of standard 3 Tesla (T) fMRI designs would have limited the scope of this approach. On the one hand, S1 activity during touch observation has been shown to be subtle, often only to survive significance thresholds when small volume corrections are applied to the fMRI data (Blakemore et al. 2005; Fitzgibbon et al. 2010; Keysers et al. 2004; Schaefer et al. 2009). This low signal strength clearly limits the possibility to characterize suppressive interactions, because subtle activity differences between different experimental conditions are of primary interest here. On the other hand, the spatial resolution of the functional data in $3 \mathrm{~T}$ fMRI studies is often too low to describe 
fine-grained architectonic characteristics in sufficient detail, for instance when specifying the topography of RFs in S1. In both cases, fMRI at ultra-high field offers a promising approach to circumvent some of these limitations (Chen et al. 2007; Kuehn et al. 2012; Stringer et al. 2011). As has recently been argued in a similar context (Kuehn et al. 2012; Stringer et al. 2011), $7 \mathrm{~T}$ fMRI can characterize brain activity changes in greater spatial detail, and with far greater sensitivity than is available with standard designs at 3 T (Bandettini 2009; Gati et al. 1997; Heidemann et al. 2012; Sanchez-Panchuelo et al. 2010; Scouten et al. 2006).

One recent study used $7 \mathrm{~T}$ fMRI to characterize S1 activity during touch observation (Kuehn et al. 2012). It was found that S1 activity during touch observation was restricted to posterior parts of contralateral $\mathrm{S} 1$; anterior parts were spared. This suggests that anterior S1 may still be considered a primary (and private) sensory brain area that is involved in social cognitive processes to a lesser extent, whereas somatosensory processes that are mediated by posterior $\mathrm{S} 1$ can be shared between, and are therefore influenced by, social interaction partners. However, that study did not characterize the functional architecture of activity changes in $\mathrm{S} 1$; it focused on the main effect of touch observation elicited by observing touch to one part of the hand. In order to characterize the topography of S1 activity during touch observation, one would have to let participants observe touch to different parts of the hands, such as different digits, and see whether distinct and topographically arranged activity changes in S1 were evoked. In addition, in order to characterize whether touch observation leads to similar inhibitory interactions between adjacent RFs, such as during physical touch perception (Gardner and Costanzo 1980; DiCarlo et al. 1998; DiCarlo and Johnson 2002; Friedman et al. 2008), one would have to compare the signal strength between conditions where touch to one finger is observed and conditions where touch to two fingers is observed, as is classically done in paradigms on suppressive interactions (Gandevia et al. 1983; Ruben et al. 2006).

For such an approach to be successful, it is important to know that single digits are distinctly represented not only in anterior $\mathrm{S} 1$, but also in posterior $\mathrm{S} 1$ (Duncan and Boynton 2007; Stringer et al. 2011; Sutherling et al. 1992), the area where touch observation elicits higher activity changes (Kuehn et al. 2012). More precisely, the $\mathrm{RF}$ of the index finger in posterior $\mathrm{S} 1$ is more lateral, more anterior, and more inferior than the RF of the middle finger of the same hand (Nelson and Chen 2008). A second important prerequisite for this approach is that, in principle, it has to be possible to characterize RF interactions by means of fMRI. Whereas many human studies have used EEG or MEG to characterize suppressive interactions between adjacently activated RFs in S1 (e.g., Biermann et al. 1998; Gandevia et al. 1983), one study with human participants successfully used fMRI for this purpose (Ruben et al. 2006). Importantly, fMRI was able to show that suppressive interactions between adjacently activated RFs in S1 occur not only in anterior but also in posterior parts of $\mathrm{S} 1$.

In the present study, we used fMRI at $7 \mathrm{~T}$ to characterize the functional architecture of S1 during touch observation along two dimensions: topographical arrangement of RFs and suppressive interactions between adjacent RFs. During the scanning session, participants observed video clips where touch was applied either to the index finger alone, the middle finger alone, or both the index and middle finger together using paintbrushes. While observing the videos, participants had to decide which of two subsequently presented paintbrushes offered the rougher stroke. This secondary task was included because the shrinkage of adjacent RFs during their concurrent activation is assumed to relate to more precise stimulus perception (Braun et al. 2002; Cardini et al. 2011; Puts et al. 2011), which we hypothesized could also transfer to the situation of touch observation. In this experiment (hereafter referred to as the observed touch experiment), no physical touch was applied to the participant's hands or fingers. Based on previous findings (Kuehn et al. 2012), we expected that particularly posterior areas of contralateral S1 would show increased activity levels when touch videos were observed. Importantly, we further expected that activity foci during observed touch to the index finger alone and middle finger alone would be partly distinct and somatotopically ordered in accordance with the expected $\mathrm{S} 1$ functional topography. We also expected that observing touch to the middle finger and to the index finger together would decrease activity levels in S1 compared with observing touch to the same fingers alone. Such a reduction could likely be explained by suppressive interactions between adjacently activated RFs.

As a control experiment (hereafter referred to as the physical touch experiment), we performed another $7 \mathrm{~T}$ fMRI experiment where physical tactile stimulation was applied to participants' index and middle fingers using paintbrushes in the same way as was observed in the observed touch experiment-the index finger alone, the middle finger alone, or both the index and middle fingers together. We expected the same pattern of results for this experiment, i.e., greater activity changes for physical touch perception compared with rest, partly distinct representations of the index and middle finger RFs in posterior $\mathrm{S} 1$, and suppressive interactions between index and middle finger RFs. In the conjunction analysis between both experiments, we expected an overlap between activity changes during physical touch perception and touch observation.

In summary, we intended to produce the first characterization of the functional architecture of S1 during touch 
observation, and relate the results to the well-described functional architecture of S1 during physical touch perception. Given the novelty of this experimental approach, our results should make an important contribution to understanding the role of S1 in social cognition.

\section{Materials and methods}

\section{Experimental design}

We conducted two separate fMRI experiments using a $7 \mathrm{~T}$ MR scanner, where participants either physically perceived tactile stimulation on their finger(s), or merely observed similar events on video. In the physical touch experiment, tactile stimulation was applied to participants' index and/or middle finger using paintbrushes. In the observed touch experiment, videos were presented to participants showing touch to the corresponding fingers, again applied using paintbrushes. The observed touch experiment was always conducted first to exclude any influence of touch experience in the scanner on touch observation (Gazzola and Keysers 2009). Both experiments were separated by 6-7 days (mean: 6.5 days \pm 1.1 days [SD]).

\section{Participants}

Sixteen healthy volunteers between 22 and 30 years (mean age 25.6 years, 8 females) participated in our study. All were right-handed (mean handedness score on the Edinburgh inventory: 98.1; Oldfield 1971), had normal or corrected-to-normal vision and none reported history of neurological, major medical, or psychiatric disorders. They were paid for their attendance and informed consent was obtained from all participants. The study was approved by the local Ethics committee at the University of Leipzig. One participant was excluded from further analyses due to a high number of missed trials $(9.4 \%)$ and a reported failure to stay awake throughout the experiment. The functional and behavioral analyses were therefore conducted with data from 15 participants.

\section{Physical touch experiment}

While subjects underwent fMRI scanning, physical tactile stimulation was applied via paintbrushes, either to the participants' middle finger (MF) or index finger (IF), or simultaneously to both the middle and index fingers (both fingers $=\mathrm{BF}$ ) of their right hands. In this way, the RF topography during physical touch perception could be described and later be compared to the RF topography during observation of touch to the corresponding fingers. In addition, added S1 activity changes during single-finger stimulation could later be compared with S1 activity changes during double-finger stimulation, as a measure of suppressive interactions (Gandevia et al. 1983; Ruben et al. 2006).

In the scanner, participants had their right hands fixed on a plastic board placed on the abdomen. The two paintbrushes used for tactile stimulation were mounted on two sticks that were connected to the plastic board to hold them at an optimal and fixed angle towards the fingers. To apply tactile stimulation, the two sticks were manually moved back and forth by the experimenter, who sat next to the scanner. Prior to the actual study, the experimenter was given intensive training in applying touch with constant pressure and with as little resultant movement as possible. Stimulation blocks lasted $24 \mathrm{~s}$, consisted of four upward and four downward strokes ( $3 \mathrm{~s}$ per stroke), and were always followed by a $24 \mathrm{~s}$ rest period. In each run, the three stimulation blocks (IF, MF, BF) were repeated three times in a randomized sequence. The experiment consisted of four runs. Thus, each stimulation block was repeated 12 times throughout the experiment, which lasted about $30 \mathrm{~min}$ in total. Participants were instructed to close their eyes and to completely relax their hands and fingers throughout the scanning session. Trial order and temporal sequence of stimulation blocks were indicated to the experimenter via earphones.

\section{Observed touch experiment}

During touch observation, participants in the scanner observed short video clips (6 $\mathrm{s}$ in duration) showing human right hands being touched by different paintbrushes. While watching the videos, no physical tactile stimulation was applied to the participants' fingers. In the videos, analogous to the physical touch experiment, either the IF, MF, or BF of the right hand were stroked by one or two paintbrushes, respectively. When only one finger was stroked, the other paintbrush stroked the tabletop next to the hand. We also included a no-touch (control) condition, where both paintbrushes stroked the tabletop, while the hand was still visible. In half of the videos, participants saw their own hand from the first person perspective; in the other half they saw another person's hand from the third person perspective. In this way, half of the observed touch and notouch videos were related to the self, whereas the other half were clearly assigned to another person. Overall, this resulted in eight experimental conditions (observed touch IF/observed touch MF/observed touch BF/no-touch $\times$ selfrelated/other-related observed touch, see Fig. 1b). Throughout the experiment, each condition was repeated 12 times.

In each trial, two brief video clips were presented in direct succession that both belonged to the same 


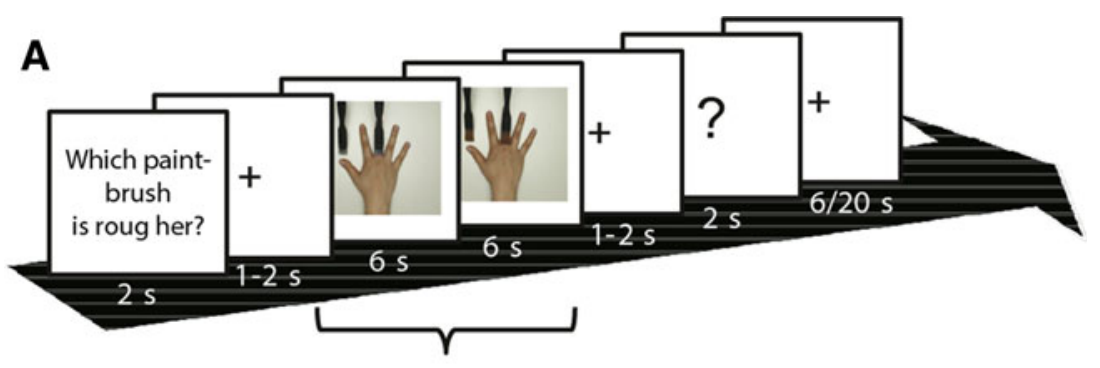

Experimental Conditions

B

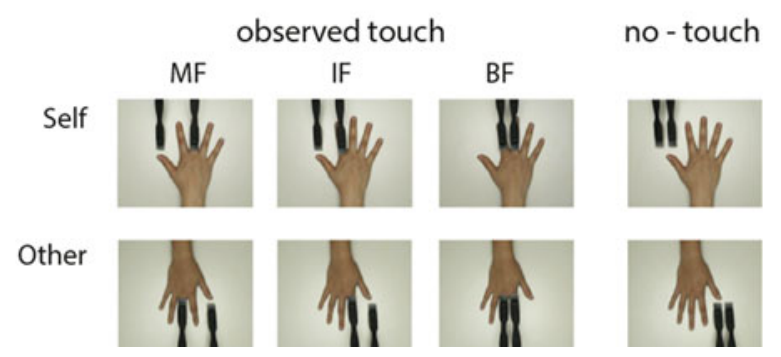

Fig. 1 Design and example trial of the observed touch experiment. a Example trial as shown to participants in the scanner. Each trial started with the same question ("Which paintbrush is rougher?") followed by two video clips presented in direct succession that belonged to the same experimental condition, but showed two different paintbrush pairs for tactile stimulation; while seeing a question mark on the screen, participants then had to indicate via lefthand button-presses which of the two paintbrush pairs was rougher. Half of the participants responded with their left index finger when they thought the first paintbrush pair was rougher and with their left

experimental condition. In both clips, the paintbrushes shown differed in their roughness levels. After watching both video clips, participants had to indicate via left hand button presses whether they thought that the first or the second video displayed the rougher paintbrushes (see Fig. 1a for an example trial). A correct response could be given in each trial (see "Stimuli" for details). This secondary task was conducted to ensure constant attention during the experiment and to estimate how well participants could judge the sensory experience related to paintbrush strokes by sight.

\section{Design}

The entire experiment took approximately $47 \mathrm{~min}$ in total and consisted of 96 trials. Half of the trials in each experimental condition showed the rougher paintbrushes first, the other half showed the smoother paintbrushes first. Trials were pseudo-randomly presented with the constraint that none of the experimental conditions was repeated more than twice in a row, and in such a way that trials in each condition added up to the same relative time point within the experiment. Prior to scanning, participants were allowed to practice the task in four trials outside the middle finger when they thought the second was rougher, the other half responded vice versa; the pause between two trials was $6 \mathrm{~s}$ in two-thirds of the trials and $20 \mathrm{~s}$ in one-third of the trials, and this was counterbalanced across conditions. b Participants saw either their own hand in the first person perspective or another person's hand in the third person perspective on video; in the observed touch conditions, touch was applied to either the middle finger (MF), the index finger (IF), or to both fingers (BF) of the right hand; in the no-touch condition, the paintbrush pair stroked the white surface on which the hand was positioned

scanner room. The experimenter ensured that they understood the task well, and were familiar with the response mode prior to entering the scanner room.

\section{Stimuli}

The video clips were recorded several weeks prior to scanning, with the same participants who later took part in the fMRI studies. During the video recordings, participants placed their right hand comfortably on a white table. Their right arm passed through a hole in a paper wall mounted in front of the table, so that they could not see their hand, the paintbrushes, or the experimenter throughout the video recordings. Their right hand was positioned such that their fingers did not touch each other but were also not stretched too far apart, and they were told to completely relax their right hand and fingers for the recording. For tactile stimulation, the experimenter used five different identicallooking paintbrushes [DaVinci paintbrushes, series 5025 (1), 5073 (2), 5036 (3), 5040 (4), and 5076 (5)]. Tactile stimulation was applied for $6 \mathrm{~s}$, either to the participants' right $\mathrm{MF}$, IF or $\mathrm{BF}$. In all conditions, two paintbrushes moved in parallel following a fixed temporal sequence that was indicated to the experimenter by an auditory signal 
(3 s per stroke, two strokes in total). All videos were recorded at a constant illumination level and with a constant angle and height between the video camera and the hands. During the video session, participants were blind with respect to the purpose of the video recordings, and they were not told how many or which kind of paintbrushes were used for stimulation.

During the fMRI experiment, the following paintbrush pairs were compared in the two videos of one trial: (1) versus (4), (2) versus (5), (1) versus (2), and (3) versus (5). Paintbrushes with higher numbers had softer and more flexible brushes and thus a smoother stroke than those with lower numbers. This was tested in a behavioral preexperiment with an independent group of 9 participants. Roughness levels of all the paintbrush pairs used could be correctly distinguished well above chance (see Online Resource 1 for more information on this experiment). As a criterion to define self/other hand pairs, we matched the self-related and other-related hands for each participant in terms of gender, and chose partners with a similar hand shape index [i.e., ratio of width to length of hand (Longo and Haggard 2010); mean hand shape difference between self/other-pairs in our study: $4.4 \%$ ]. Importantly, once a self/other hand pair was identified, the videos that served as the self conditions for one partner always served as the other conditions for the matching partner. In this way, self/ other differences in the video clips that could not be explicitly controlled (for example differences in skin color) were counterbalanced across participants.

Imaging acquisition parameters

Functional and structural MRI data were acquired using a 7 T MR scanner (Magnetom 7T, Siemens Healthcare Sector, Erlangen, Germany) with a 24-channel NOVA head coil. Prior to both experiments, high-resolution 3D anatomical T1-weighted scans were acquired. For the observed touch experiment, which was always conducted first, structural data were acquired using the MP2RAGE sequence with the following parameters: $\mathrm{TR}=5.0 \mathrm{~s}$, $\mathrm{TE}=2.45 \mathrm{~ms}$, TI1/TI $2=900 \mathrm{~ms} / 2,750 \mathrm{~ms}$, flip angle $1 /$ flip angle $2=5^{\circ} / 3^{\circ}$ with an isotropic voxel resolution of $0.7 \mathrm{~mm}$ (Marques et al. 2010). For the physical touch experiment, we used a shorter T1-sequence with a slightly reduced spatial resolution, because the T1-images acquired for each participant during the observed touch experiment could later be used for data analyses; the MP2RAGE sequence here served only to anatomically localize $\mathrm{S} 1$ in the subsequently measured functional scans. Acquisition parameters in the physical touch experiment were: $\mathrm{TR}=$ $4.0 \mathrm{~s}, \quad \mathrm{TE}=2.36 \mathrm{~ms}$, TI $1 / \mathrm{TI} 2=900 \mathrm{~ms} / 2,750 \mathrm{~ms}$, flip angle $1 /$ flip angle $2=5^{\circ} / 3^{\circ}$ with an isotropic voxel resolution of $0.9 \mathrm{~mm}$. Shimming was performed in both experiments prior to collecting the functional data. In both experiments, T1-weighted scans were subsequently used to select 30 axial slices (interleaved slice acquisition, slice thickness $=1.5 \mathrm{~mm}$, no gap) covering bilateral $\mathrm{S} 1$ and adjacent areas (see Fig. 2). The hand knob area was used for this purpose. This is easily identified in sagittal T1-images, and reliably indicates the location of the hand area in the primary motor cortex (Yousry et al. 1997), and the primary somatosensory cortex (Moore et al. 2000; Sastre-Janer et al. 1998; White et al. 1997). Functional T2*-weighted gradientecho echo-planar images were then acquired using GRAPPA acceleration (iPAT $=3$; Griswold et al. 2002). A field of view of $192 \times 192 \mathrm{~mm}^{2}$ and an imaging matrix of $128 \times 128$ were used. The functional images had isotropic $1.5 \mathrm{~mm}$ voxels. The other sequence parameters were: $\mathrm{TR}=1.5 \mathrm{~s}, \mathrm{TE}=20 \mathrm{~ms}$, flip angle $=90^{\circ}$. The acquisition parameters of the functional scans were identical for the physical and observed touch experiments.

To attenuate scanner noise, participants were provided with earplugs and ear-defenders. During the observed
Fig. 2 Selected slices for functional imaging of one example subject. Shown is a sagittal slice of the anatomical MP2RAGE scan which was used to select 30 axial slices covering bilateral $\mathrm{S} 1$ on the basis of the individual subject's brain anatomy (i.e., hand knob area)

\section{Acquisition of functional images}

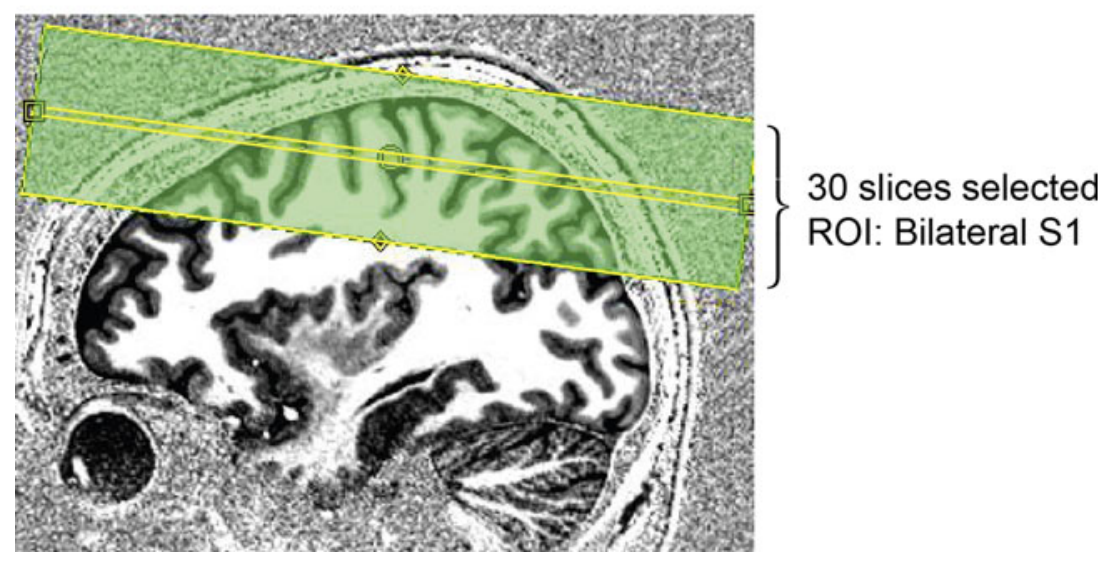


touch experiment, the middle and index fingers of participants' left hands were placed on two buttons of a response box. Visual stimuli were projected onto a plastic screen vertically mounted in front of the participants, which could be looked at via a mirror mounted on the receiver coil.

\section{fMRI preprocessing and localization of S1 activity}

Preprocessing and statistical analyses of the functional imaging data were carried out using SPM8 (Statistic Parametric Mapping, Wellcome Department of Imaging Neuroscience, University College London, London, UK). A slice timing correction was applied to correct for differences in image acquisition time between slices, and realignment was performed to minimize movement artifacts in the time series (Unser et al. 1993a, b). Normalization to standard MNI space was done using the unified segmentation approach based on image registration and tissue classification (Ashburner and Friston 2005). The high-resolution T1-weighted images of both sessions were used to visually confirm correct registration between the sessions. In addition, the co-registration of each participant within the experiments was visually checked in order to identify possible spatial distortion effects that may occur at higher field strength (none were found, however). Data were filtered with a high-pass filter of $0.01 \mathrm{~Hz}$ to eliminate slow signal drifts. Data were smoothed with a Gaussian kernel of $4 \mathrm{~mm}$ full-width half-maximum (FWHM).

$\mathrm{S} 1$ is not a homogenous area, but can be classified into four sub-areas (from rostral to caudal: areas $3 \mathrm{a}, 3 \mathrm{~b}, 1$ and 2). The Anatomy Toolbox implemented in SPM8 (Eickhoff et al. 2005, 2006, 2007; Geyer et al. 1999, 2000; Grefkes et al. 2001) was used to specify in which sub-area activity changes took place for the normalized group-level and single-subject analyses. Single-subject analyses were additionally performed without normalization into stereotactic space in order to describe the site of the suppressive interaction effect as precisely as possible in relation to the cortical anatomy of each individual subject. We used guidelines that linked cytoarchitectonic labeling with anatomical descriptions of subregions (Geyer et al. 1999, 2000; Grefkes et al. 2001; White et al. 1997). According to these specifications, areas $3 \mathrm{a}$ and $3 \mathrm{~b}$ (anterior S1) are found in the deep valley of the central sulcus and in the anterior wall of the postcentral gyrus, respectively, whereas areas 1 and 2 (posterior $\mathrm{S} 1$ ) are located at the crown of the postcentral gyrus and at the posterior wall of the postcentral gyrus, respectively. It is important to note that although these specifications are useful, no clear anatomical landmark exists for the exact transition zone between S1 subregions (Geyer et al. 1999). However, using a combination of automated and manual labeling, and by including singlesubject analyses, the localization of the suppressive interaction effect in $\mathrm{S} 1$ in the current study is described in reasonable detail.

fMRI statistical analyses

A general linear model (GLM) was fitted to the data and $t$-maps were created on the individual subject level. In the observed touch experiment, the observation times of the two video sequences in each trial were modeled as blocks and used to compute contrast images by linear combination of parameter estimates. In the physical touch experiment, the physical tactile stimulation blocks were used for this same purpose. In the observed touch experiment, the questions and the button-press events were included into the model as regressors. We used one-sample $t$ tests at the second level to calculate different contrasts for the physical and observed touch experiments. All contrasts on the group level and on the normalized singlesubject level were a priori masked with the anatomical S1 mask offered by the Anatomy Toolbox implemented in SPM8. Because the functionally scanned regions were manually selected in each individual subject based on the T1 scans (see Fig. 2), thus always covering S1 but covering varying parts of the motor or parietal cortices depending on the individual subject's brain anatomy, this anatomical masking ensured comparable data analyses for all participants.

For the group-level calculations, reported voxels of the functional data were considered significant at $p<0.001$ when belonging to a cluster significant at $p<0.05$ (FWEcorrected). In addition, we describe some of the grouplevel clusters at uncorrected cluster-thresholds (i.e., without FWE-correction) when this provided additional information (for example on the question of whether ipsilateral S1 would show any sub-threshold activity). When these more liberal analyses were used, it is explicitly pointed out in the results section. In addition to group-level analyses, single-subject analyses were also performed in order to describe some of the reported effects in greater detail. For the single-subject analyses, reported voxels were thresholded at $p<0.001$. They are mentioned when belonging to a cluster with a minimum size of five voxels $\left(16.8 \mathrm{~mm}^{3}\right)$.

\section{Topographical arrangement}

For the physical touch experiment, we calculated the main effect of physical touch perception versus rest (IF touch $+\mathrm{MF}$ touch $+\mathrm{BF}$ touch $-3 \times$ rest), and the specific effects of touch applied only to the IF, MF, and to BF, respectively (e.g., IF touch - rest). For the observed touch experiment, we calculated the main effect of observing touch to the hand versus observing no touch to the hand, as presented in the videos [(self-related observed touch $\mathrm{IF}+$ self-related observed touch $\mathrm{MF}+$ self-related observed touch $\mathrm{BF}-3 \times$ no-touch self) + (other-related 
observed touch IF + other-related observed touch $\mathrm{MF}+$ other-related observed touch $\mathrm{BF}-3 \times$ no-touch other)]. This effect was also calculated separately for observing touch to the IF, MF and BF, respectively. To look at whether S1 RFs during observed touch were topographically aligned, we masked the observed touch contrasts with the corresponding physical touch masks (e.g., observed touch IF - no-touch masked with IF touch - rest) and non-corresponding physical touch masks (e.g., observed touch IF - no-touch masked with MF touch - rest). Physical touch masks contained all voxels significant at $p<0.001$ that belonged to a cluster significant at $p<0.05$ (FWE-corrected). In addition, we estimated whether the topographical arrangement of S1 RFs during observed touch followed the expected pattern (i.e., the RF of the IF was supposed to be more lateral, more anterior, and more inferior than the RF of the MF).

\section{Suppressive interactions}

To calculate suppressive interactions between adjacent RFs in $\mathrm{S} 1$, we compared the expected activity changes to the actual activity changes during BF physical stimulation or observation, respectively. More precisely, for the physical touch experiment, we calculated the expected activity changes in S1 in the case where signal changes during touch applied to the IF and MF added up linearly. This was calculated as a first-level contrast [i.e., (IF touch - rest) + (MF touch - rest)]. This contrast was then compared with the actual activity changes during BF stimulation (i.e., BF touch - rest). Also this calculation was performed at the first-level. Note that this is analogous to how suppressive interactions during physical touch were characterized in a previous fMRI study (Ruben et al. 2006), as we confirmed in an additional analysis (results not reported).

For the observed touch experiment, analogous methodology was applied: to measure suppressive interactions during observed touch, we first calculated the expected activity changes in S1 if activity changes during observed touch to the IF and MF added up linearly [i.e., (self-related observed touch MF + self-related observed touch IF $-2 \times$ no-touch self) + (other-related observed touch $\mathrm{MF}+$ other-related observed touch IF $-2 \times$ no-touch other)]. To define suppressive interactions during observed touch, this contrast was compared with actual S1 activity changes during observed touch to BF [i.e., (self-related observed touch $\mathrm{BF}-$ no-touch self) + (other-related observed touch BF - no-touch other)]. These same analyses were also performed separately for the self- and other-related observed touch conditions.

The suppressive interaction contrast therefore specified signal decreases in S1 that particularly occur in the BF stimulation and observation conditions, respectively.
Signal decreases in these conditions that most likely reflect suppressive interactions are those which occur in voxels that belong to the RF of one of the two single fingers. Other signal drops can be less easily explained by suppressive interactions between adjacently activated RFs. We, therefore, spatially specified the suppressive interaction contrast by only describing significant activity decreases in voxels that that belonged to the RFs either of the IF or of the MF. More precisely, the suppressive interaction contrast for physical touch perception was masked by all voxels that were activated by physical IF stimulation plus those that were activated by physical MF stimulation at $p<0.001$ belonging to a cluster of $p<0.05$ (FWE-corrected; see Ruben et al. 2006 for a similar approach). The suppressive interaction effect for observed touch was analogously masked by all voxels that were activated during observed touch to the IF plus those that were activated during observed touch to the MF. Here, we restricted our search volume to this mask area. Note that this spatial specification was necessary in order to clearly identify signal decreases that were specific to the effect under investigation (i.e., suppressive interactions).

We also needed a way of quantifying the degree of suppressive interactions, both during physical touch perception and during touch observation. Using contrast estimates, we calculated interaction ratios (IRs), which have frequently been used to specify the relation between expected and real activity changes in $\mathrm{S} 1$ (IR $=100-$ $([\mathrm{BF} /(\mathrm{IF}+\mathrm{MF})] \times 100)$; Biermann et al. 1998; Hsieh et al. 1995; Ishibashi et al. 2000; Ruben et al. 2006). We used the masks created for specifying the suppressive interaction contrast for physical touch and observed touch (see previous paragraph) to extract all relevant contrast estimates for each individual subject. The individual IRs could then be used to calculate the mean IR across participants. This allows a much more detailed and spatially specific analysis than taking the contrast estimates as a mean across the whole group of participants. However, this procedure comes with the cost of only allowing the analysis of those participants for whom a mask could be created. More precisely, only those participants who showed significant activity changes at the single-subject level for physically/visually perceiving touch to the MF and IF could be included. As expected, this was the case for all participants with respect to physical touch perception $(N=15)$. However, this was not the case for all participants with respect to touch observation. More precisely, $n=10$ participants could be used to calculate the mean IR for observed touch, and $n=9$ participants could be used to calculate the mean IR for self-related observed touch. For other-related observed touch, only $n=4$ participants fulfilled these criteria (i.e., $n=11$ participants did not show significant activity changes when observing other-related 
touch to the MF or to the IF at the single-subject level). Thus, the mean IR for other-related observed touch was not calculated.

To summarize, whereas voxel-wise statistics included all participants $(N=15)$, contrast estimates to calculate the IR during observed touch could only be extracted for a subset of participants due to the masking procedure that required significant single-subject results $[n=10$ for main effect of observed touch, $n=9$ for self-related observed touch, $n=4$ for other-related observed touch (not calculated)].

\section{Overlapping RFs}

To find out whether suppressive interactions occurred only in voxels where IF and MF RFs overlapped (and could, thus, theoretically be explained by ceiling effects of the BOLD signal, for example), or also occurred in nonoverlapping voxels, we additionally calculated whether suppressive interactions occurred only in "overlapping", or also in "non-overlapping" S1 voxels. Overlapping voxels were defined as those voxels in S1 that were active when both touch to the IF and touch to the MF were observed (i.e., observed touch IF - no-touch $\cap$ observed touch $\mathrm{MF}$ - no-touch) or experienced (IF touch - rest $\cap \mathrm{MF}$ touch - rest), whereas non-overlapping voxels were those which did not overlap between the two contrasts. We calculated the percentage (\%) of suppressive interaction voxels in either category (i.e., suppressive interaction effect in overlapping and non-overlapping voxels) separately for physical and observed touch.

\section{Behavioral analyses}

During the observed touch experiment, participants solved a secondary two-alternative forced-choice task in the scanner, in which they had to indicate which of two subsequently presented video clips displayed the rougher paintbrush pair. The two video clips presented in one trial always showed paintbrushes of different roughness levels, such that a correct or incorrect response could be given in each trial. We performed a repeated-measures analysis of variance (ANOVA) to estimate the influence of hand identity (self-related, other-related) and observed event (observed touch, no-touch) on the percentage of correct responses given in this task. We also calculated a one-way ANOVA to estimate the influence of finger touch (MF, IF, $\mathrm{BF}$ ) on the percentage of correct responses. To investigate whether the individual degrees of suppressive interactions across trials were related to how precisely roughness levels could be estimated by sight, we performed Pearson correlations between individual IRs during touch observation and the percentage of correct responses both for the IRs across conditions and the IRs for the self-conditions.

\section{Results}

Topographical arrangement

As expected, physical touch administered to participants' right fingers activated, as a main effect, a large significant cluster in left (contralateral) S1 that peaked in left posterior S1, and extended to left anterior S1. Touch applied specifically to the right IF, MF, or to BF, respectively, also activated significant focal areas in left S1. The IF and MF RFs partly overlapped, but were also partly distinct. The significant clusters peaked in left posterior S1, but extended to left anterior S1 (see Fig. 3a; Table 1). No significant activity changes were found in right (ipsilateral) S1 for these contrasts. We also looked at sub-threshold activity in ipsilateral S1. Here, we found that when the significance threshold of $p<0.001$ and $k \geq 5$ was not FWE-corrected, one cluster in right (ipsilateral) $\mathrm{S} 1$ showed greater activity during physical touch perception compared with rest $[k=9 ; t=4.05 ; 53$, $-18,34(x, y, z)$ ] (see Online Resource 2 for a complete list of sub-threshold activity changes).

For observed touch, we found that looking at a hand being touched compared with looking at the same hand not being touched significantly increased activity in left (contralateral) posterior S1. Note that participants did not receive any tactile stimulation in either of these observation conditions. No significant activity changes were found for the reverse contrast (no-touch vs. observed touch). In addition, we were interested in whether right (ipsilateral) S1 would show any sub-threshold activity during touch observation. When we omitted the FWE-correction, significant activity changes in ipsilateral $\mathrm{S} 1$ were found $(p<0.001$ and $k \geq 5)$. This cluster was localized in posterior parts of right $\mathrm{S} 1$ (see Online Resource 2 for a list of all sub-threshold activity changes).

Observing touch to specific fingers also activated left (contralateral) S1. Whereas activity changes in left S1 during observed touch to the IF and to BF survived the standard cluster-corrected thresholds, activity change in left S1 during observed touch to the MF was only significant when no cluster-correction was applied $(p<0.001$ and $k \geq 5$ ). All observed touch clusters peaked in left posterior S1 (see Table 1; Fig. 5). To verify that activity changes in contralateral S1 in response to touch observation were restricted to posterior S1, and did not occur in anterior S1 (particularly in area 3b), we conducted an ROI analysis focusing on left area $3 \mathrm{~b}$. We masked the contrast observed touch-no-touch with the left area $3 \mathrm{~b}$ mask provided by the Anatomy toolbox implemented in SPM. Here, 


\section{APhysical Touch}
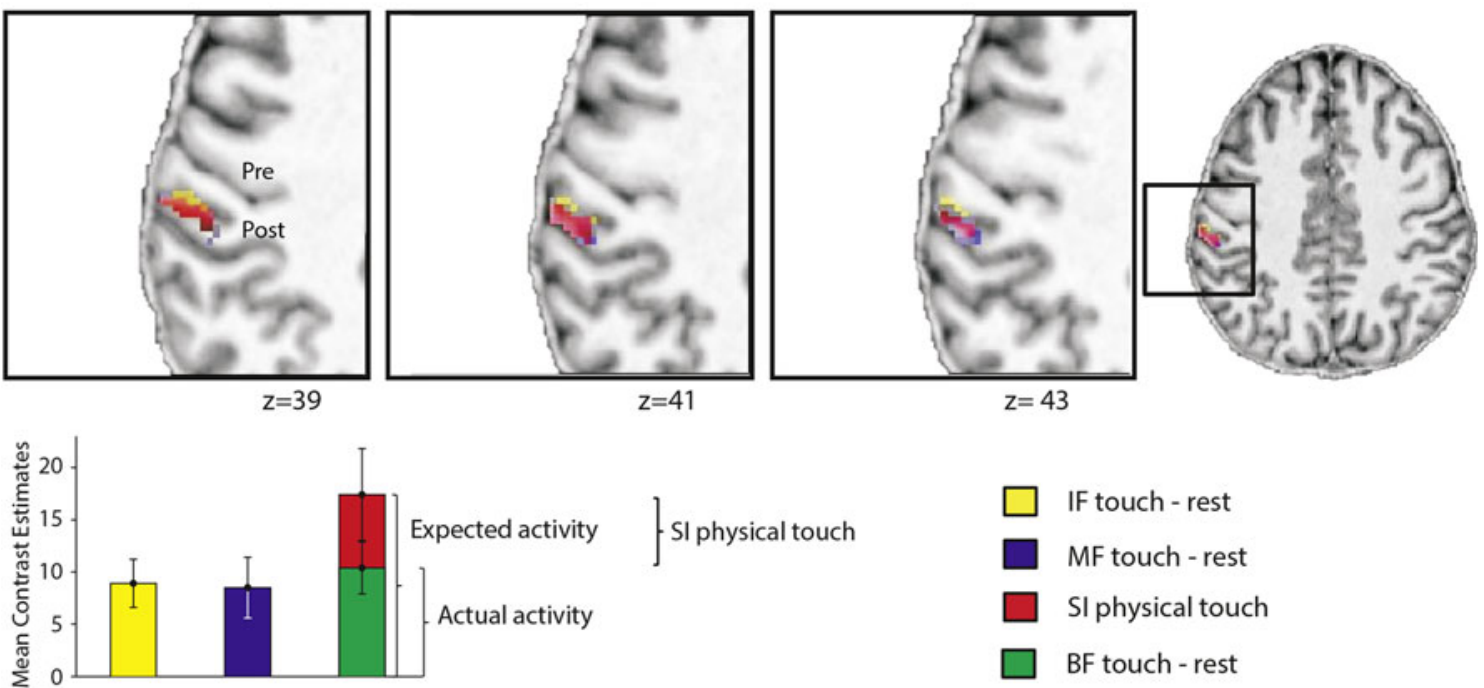

IF touch - rest

$\square$ MF touch - rest

$\square$ SI physical touch

$\square$ BF touch - rest

\section{BObserved Touch}
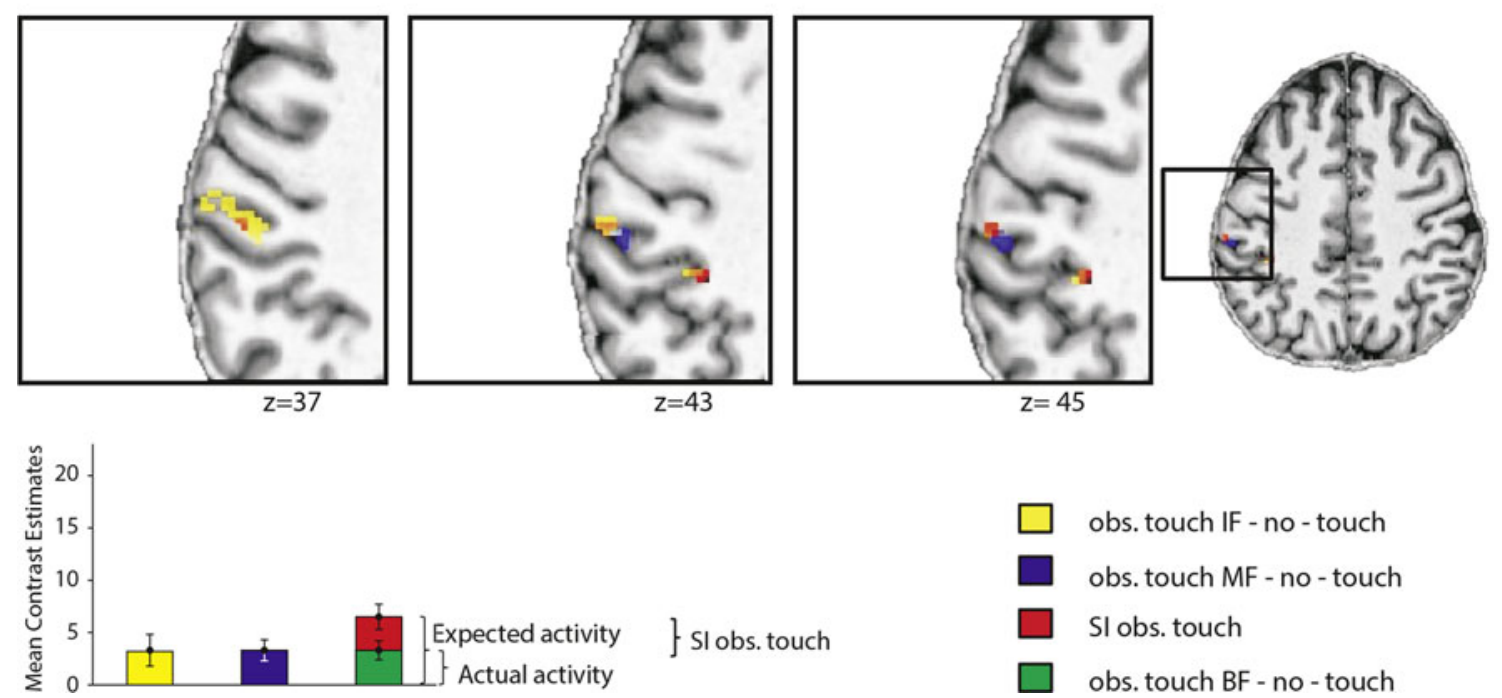

Fig. 3 Suppressive interactions (SI) in contralateral S1 during physical touch perception (a) and touch observation (b). a Activity changes of contralateral S1 during physical touch applied to the index finger (IF) and the middle finger (MF); in addition, the suppressive interaction (SI) effect for physical touch is displayed using voxel-wise statistics in the upper panel (IF touch-rest + MF touch-rest - BF touch-rest), and using contrast estimates in the lower panel; the bar labeled "Expected activity" describes the added contrast estimates of physical touch to the IF and MF, whereas the bar labeled "Actual activity" describes the contrast estimates when both fingers were stimulated together; the bar graphs show mean contrast estimates \pm standard deviation (SD) of all $(N=15)$ participants. b Activity changes of contralateral S1 during observed touch to the IF and MF; in addition, the SI effect for observed touch is displayed

we found that no significant activity changes survived the standard significance threshold, even when voxels at $p<0.001$ and $k \geq 5$ belonging to uncorrected clusters were taken into account. using voxel-wise statistics in the upper panel (obs. touch IF-notouch + obs. touch MF-no-touch - obs. touch BF-no-touch), and using contrast estimates in the lower panel; the bar labeled "Expected activity" describes the added contrast estimates of touch observation to the IF and MF, whereas the bar labeled "Actual activity" describes the contrast estimates when touch to both fingers together was observed; the bar graphs show mean contrast estimates \pm standard deviation (SD) of $n=10$ participants (see "Suppressive interaction" for details on why not all participants were part of this analysis); functional images are masked with an anatomical mask covering contralateral S1 and are thresholded at $p<0.0005$ (uncorrected) (a) and $p<0.001$ (uncorrected) (b); the data are displayed on a normalized T1-image of an individual subject; Pre precentral gyrus, Post postcentral gyrus

We also looked at whether S1 activity changes during observed touch overlapped with S1 activity changes during physical touch. We masked the contrast observed touchno-touch with physical touch-rest, and found that 
Table 1 S1 activity changes during physical touch perception and touch observation in different experimental conditions
Listed clusters contain voxels thresholded at $p<0.001$ and are cluster-corrected at $p<0.05$ (FWE-corrected); the two contrasts which are marked with a \# show clusters that are not cluster-corrected, but contain a minimum of five voxels using the same voxel threshold; see Online Resource 2 for a complete list of sub-threshold activity changes for all listed contrasts

obs. observed, $M F$ middle finger, $I F$ index finger, $B F$ both fingers, $S I$ suppressive interactions, $I P C$ inferior parietal cortex

\begin{tabular}{|c|c|c|c|c|}
\hline Contrast & Area & $\begin{array}{l}\text { MNI location } \\
(x, y, z)\end{array}$ & $\begin{array}{l}\text { Peak } \\
t \text { value }\end{array}$ & $\begin{array}{l}\text { No. of } \\
\text { voxels }\end{array}$ \\
\hline \multicolumn{5}{|l|}{ Physical touch } \\
\hline $\mathrm{MF}$ touch $+\mathrm{IF}$ touch $+\mathrm{BF}$ touch-rest & L Area 2 & $-55-2440$ & 8.31 & 685 \\
\hline \multirow[t]{2}{*}{ MF touch-rest } & L Area 2 & $-55-2440$ & 7.33 & 185 \\
\hline & L Area 2 & $-44-3357$ & 6.78 & 215 \\
\hline IF touch-rest & L Area 2 & $-37-3963$ & 9.03 & 562 \\
\hline BF touch-rest & L Area 2 & $-56-2242$ & 9.37 & 991 \\
\hline SI physical touch & L Area 2/L IPC & $-54-2639$ & 7.47 & 123 \\
\hline \multicolumn{5}{|l|}{ Observed touch } \\
\hline \multirow{2}{*}{$\begin{array}{l}\text { Obs. touch } \mathrm{MF}+\text { obs. touch IF } \\
+ \text { obs. touch } \mathrm{BF}-\text { no-touch }\end{array}$} & L Area 2 & $-37-4454$ & 4.35 & 245 \\
\hline & L Area 2 & $-55-2444$ & 4.04 & 179 \\
\hline \multirow[t]{4}{*}{ \# Obs. touch MF - no-touch } & L Area 1 & $-26-5466$ & 5.02 & 6 \\
\hline & L Area 2 & $-26-5157$ & 5.00 & 17 \\
\hline & L Area 2 & $-54-2745$ & 3.45 & 19 \\
\hline & L Area 1 & $-60-1836$ & 3.29 & 9 \\
\hline Obs. touch IF - no-touch & L Area 2 & $-58-2140$ & 6.05 & 139 \\
\hline \multirow[t]{2}{*}{ Obs. touch BF - no-touch } & L Area 2 & $-34-4457$ & 6.27 & 127 \\
\hline & L Area 2 & $-54-2444$ & 5.56 & 128 \\
\hline \multirow[t]{2}{*}{ SI observed touch } & L Area 2 & $-55-2646$ & 4.54 & 8 \\
\hline & L Area 2 & $-36-3644$ & 4.51 & 5 \\
\hline SI observed touch self & L Area 2 & $-40-4262$ & 4.36 & 9 \\
\hline \multicolumn{5}{|l|}{ Observed touch $\cap$ Physical touch } \\
\hline $\begin{array}{l}\text { Obs. touch } \mathrm{MF}+\text { obs. touch } \mathrm{IF} \\
+ \text { obs. touch } \mathrm{BF}-\text { no-touch } \cap \mathrm{MF} \text { touch } \\
+ \text { IF touch }+\mathrm{BF} \text { touch-rest }\end{array}$ & L Area 2 & $-55-2444$ & 5.70 & 162 \\
\hline \multirow[t]{2}{*}{ \# Obs. touch MF-no-touch $\cap$ MF touch-rest } & L Area 2 & $-54-2745$ & 4.45 & 15 \\
\hline & L Area 1 & $-61-1633$ & 4.15 & 10 \\
\hline Obs. touch IF-no-touch $\cap$ IF touch-rest & L Area 2 & $-58-2140$ & 6.05 & 123 \\
\hline Obs. touch BF-no-touch $\cap \mathrm{BF}$ touch-rest & L Area 2 & $-54-2444$ & 5.56 & 114 \\
\hline
\end{tabular}

mask would have any significant effect on the number of voxels included in the corresponding and non-corresponding masks, we performed a Chi-square test including the number of voxels significant within the two mask conditions of both contrasts. This test did not reach statistical significance $(p>0.2)$. When estimating the percentage of participants who showed overlapping activity changes between physical and observed touch in the different conditions, we found that $75 \%$ of participants showed shared voxels for observed touch to the IF, $78 \%$ showed shared voxels for observed touch to the MF, and $92 \%$ showed shared voxels for observed touch to BF.

We additionally looked at the topographic arrangement of activity changes as evoked by observing touch to the IF and the MF, respectively. We found that activity changes in both conditions were partly overlapping, but partly distinct. Importantly, activity changes evoked by observing touch to the IF were more lateral, more anterior, and more inferior 
Fig. 4 Overlap between activity changes during physical touch perception and touch observation in contralateral S1. Overlapping voxels between physical touch perception and touch observation are displayed in purple; overlaps are shown separately for the index finger (upper panel) and the middle finger (lower panel); functional images are masked with an anatomical mask covering contralateral S1 and thresholded at $p<0.001$ (uncorrected); functional data are visualized on a normalized T1 image of an individual subject; Pre precentral gyrus, Post postcentral gyrus
Index Finger Overlap
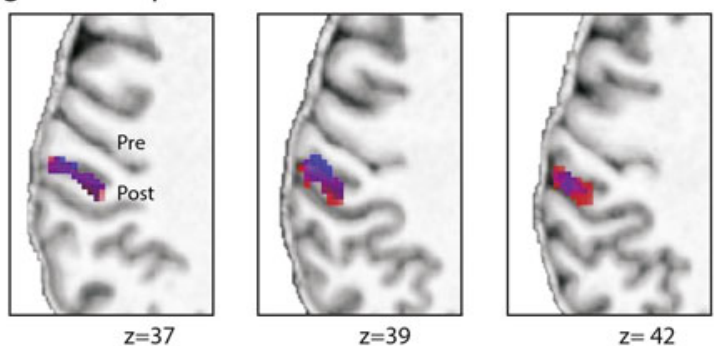

Middle Finger Overlap
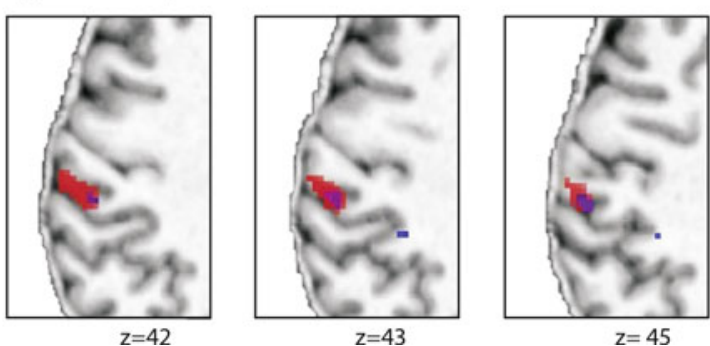

physical touch - rest

obs. touch - no - touch

physical touch - rest $n$ obs. touch - no - touch than activity changes evoked by observing touch to the MF (see Table 1; Fig. 5).

One last analysis was performed due to a concern that activity changes in contralateral S1 during touch observation could be explained by preparatory motor activity for the later button-press responses rather than by touch observation. To counter this argument, we looked at whether left and right primary motor cortex (M1) showed any increased activity changes during touch observation that could indicate preparatory motor activity or motor imaginary during touch observation. There were no significant activity changes neither in left nor in right M1 for the observed touch-no-touch contrast. This was also true when not correcting for multiple comparisons.

\section{Suppressive interactions}

In order to estimate the degree of suppressive interactions in S1 during physical touch perception, we compared the summed activity changes during physical touch perception to the IF and MF to the activity changes in the BF stimulation condition. We found a significant suppressive interaction effect for physical touch in left (contralateral) S1, which peaked at the border between left area 2 and the left inferior parietal cortex, and extended to left area 2, left BA 1, and left area $3 b$ (see Table 1; Fig. 3a). No significant suppressive interaction effect was found in right (ipsilateral) S1, even when the analysis was performed without correcting for multiple comparisons. At the individual subject level, suppressive interactions for physical touch were significant in all but one of the investigated participants $(n=14)$, and could be assigned to left area 1 and left area 2. Only a subset of participants showed additional significant activity changes in left area $3 \mathrm{~b}(n=4)$ and left area $3 \mathrm{a}(n=2)$ for this contrast. The mean IR for physical touch perception was $37.2 \%, \mathrm{SD}=15.9$ (Fig. 3a, see Fig. 6 for single subject data).

We then calculated the suppressive interaction effect for observed touch, which was similarly calculated by comparing summed activity changes in $\mathrm{S} 1$ evoked by observing touch to two single fingers separately to activity changes evoked by observing touch to both fingers together. Here, we found a significant suppressive interaction effect in two clusters that both peaked in left (contralateral) area 2. One cluster also extended to left area 1, the other extended to the left superior parietal lobule (see Table 1; Fig. 3b). No significant suppressive interaction effect was found for right (ipsilateral) S1. At the individual subject level, we found a significant suppressive interaction effect in left S1 for $n=8$ participants (see individual subject data of $n=5$ participants in Fig. 6). In all of them, the effect was located in left area 2. In $n=7$ participants, activity changes also extended to left area 1, and in one subject, the activity changes extended to left area 3. Note that most of the other participants also showed suppressive interaction voxels in left S1 during observed touch, but these results are not reported due to the relatively conservative single-subject threshold we defined for our analyses [e.g., $n=14$ participants showed a suppressive interaction effect when we lower the single-subject threshold to $p<0.005$ (uncorrected)].

We also calculated the suppressive interaction effect specifically for self- and other-related observed touch. The suppressive interaction effect for self-related observed touch revealed one significant cluster in left area 2 . The suppressive interaction effect for other-related observed 
Fig. 5 Receptive field (RF) topography of the index finger (IF) and middle finger (MF) in contralateral S1 during physical touch perception and touch observation. Shown are five axial slices ordered from inferior $(z=42)$ to superior $(z=47)$ of $N=15$ participants; the borders of the MF RFs are indicated using blue lines; functional images are masked with an anatomical mask covering contralateral S1 and visualized at an individual's normalized T1 image; to make both conditions better comparable, a slightly more conservative threshold was chosen for physical touch perception $[p<0.0001$ (uncorrected)] than for touch observation $[p<0.001$ (uncorrected)]
RF Topography Physical Touch
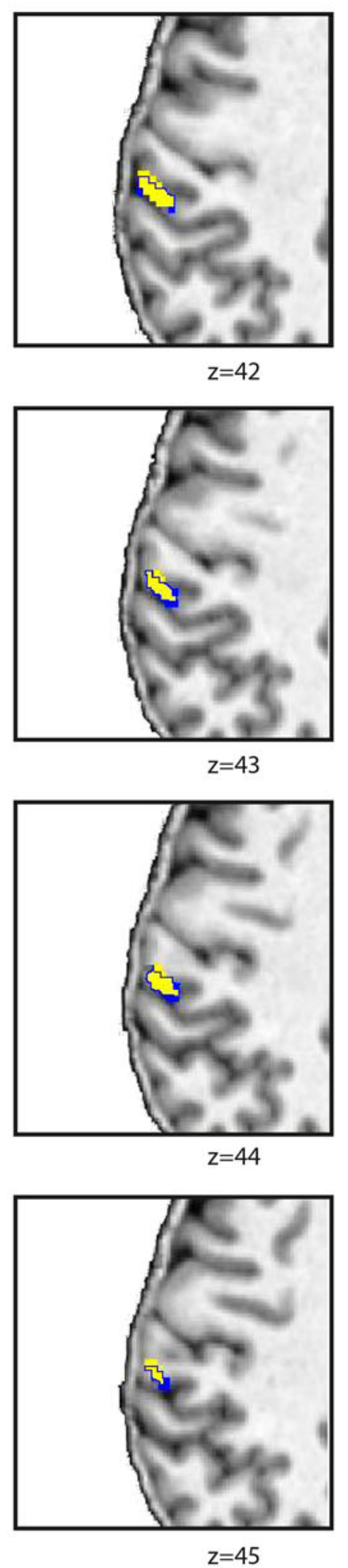

IF touch - rest

MF touch - rest

\section{RF Topography Observed Touch}

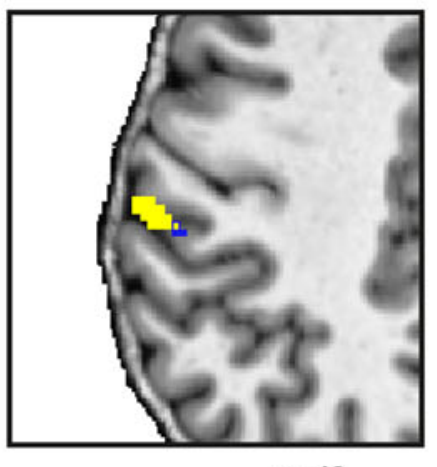

$z=42$

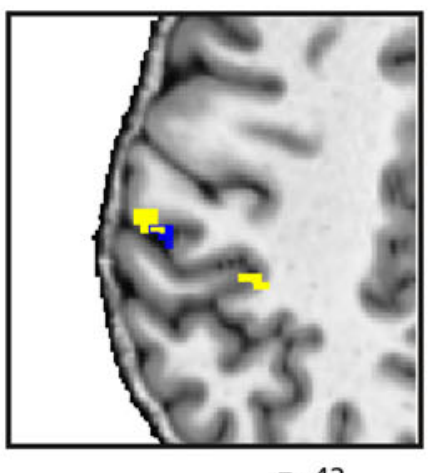

$z=43$

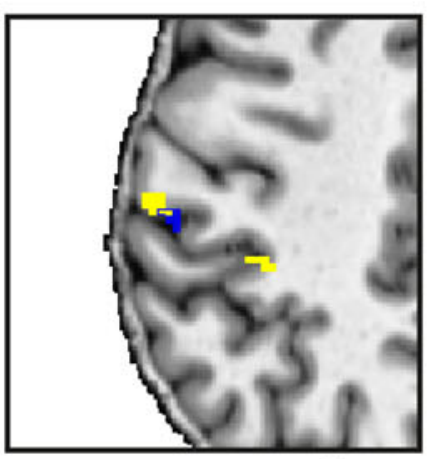

$z=44$

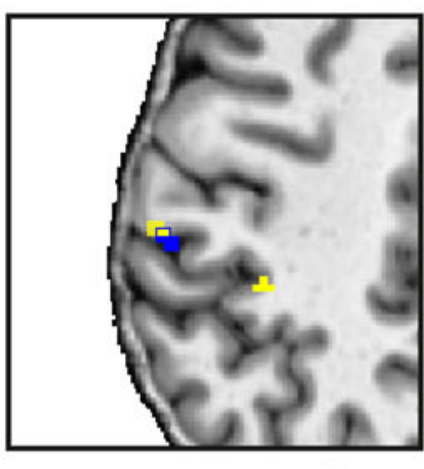

$z=45$

obs. touch IF - no - touch

obs. touch MF - no - touch 


\section{Physical Touch}

Sub. 1

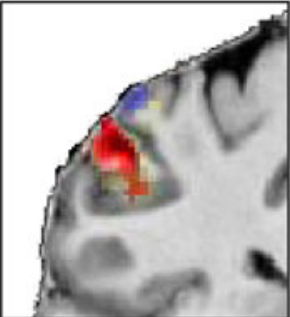

$y=-28$

Sub. 2

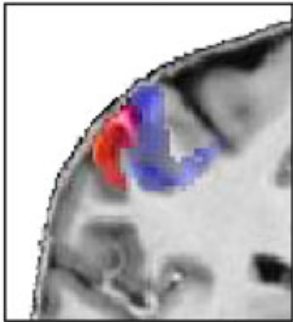

$y=-28$

Sub. 3

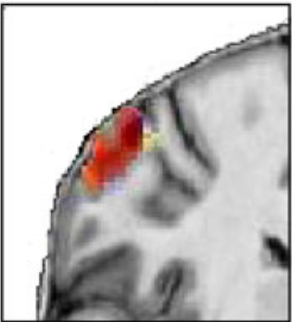

$y=-20$

Sub. 4

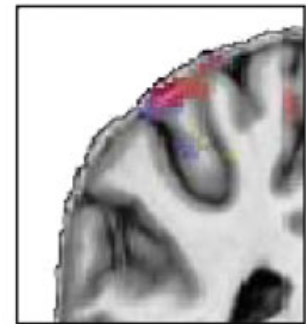

$y=-40$

Sub. 5

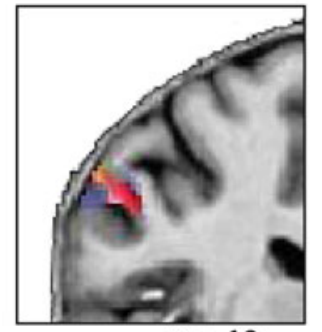

$y=-18$

IF touch - rest

MF touch - rest

SI physical touch

Fig. 6 Contralateral S1 activity during physical touch perception and touch observation of $n=5$ individual subjects. The left side of the figure shows functional data of physical touch to the index finger (IF), the middle finger (MF), and the suppressive interaction (SI) effect for physical touch; the right side of the figure shows functional data of observed touch to the IF, the MF, and the SI effect for observed touch;

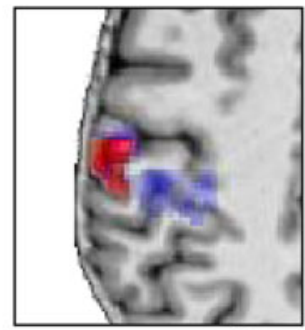

$z=44$
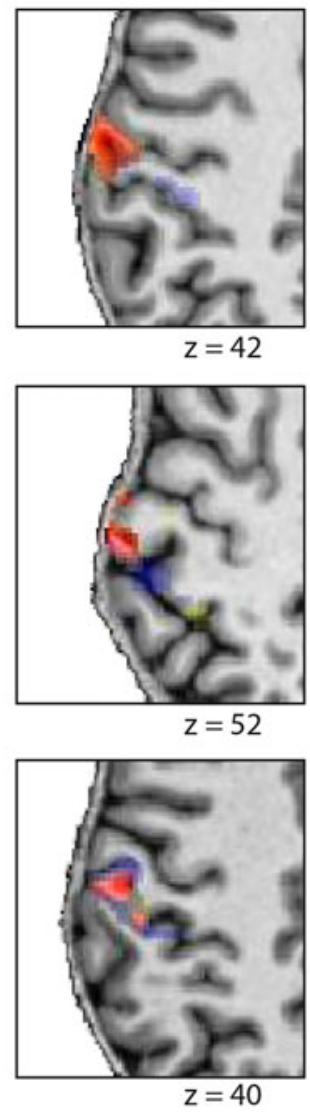

\section{Observed Touch}

Sub. 1

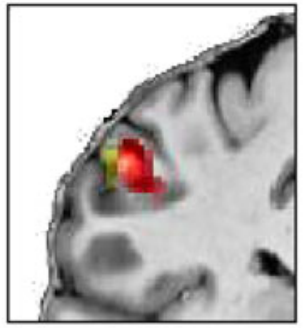

$y=-28$

Sub. 5

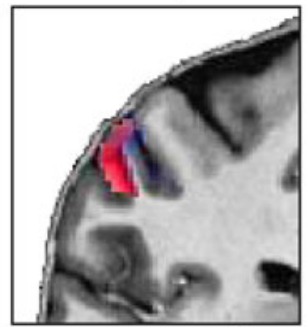

$y=-28$

Sub. 3
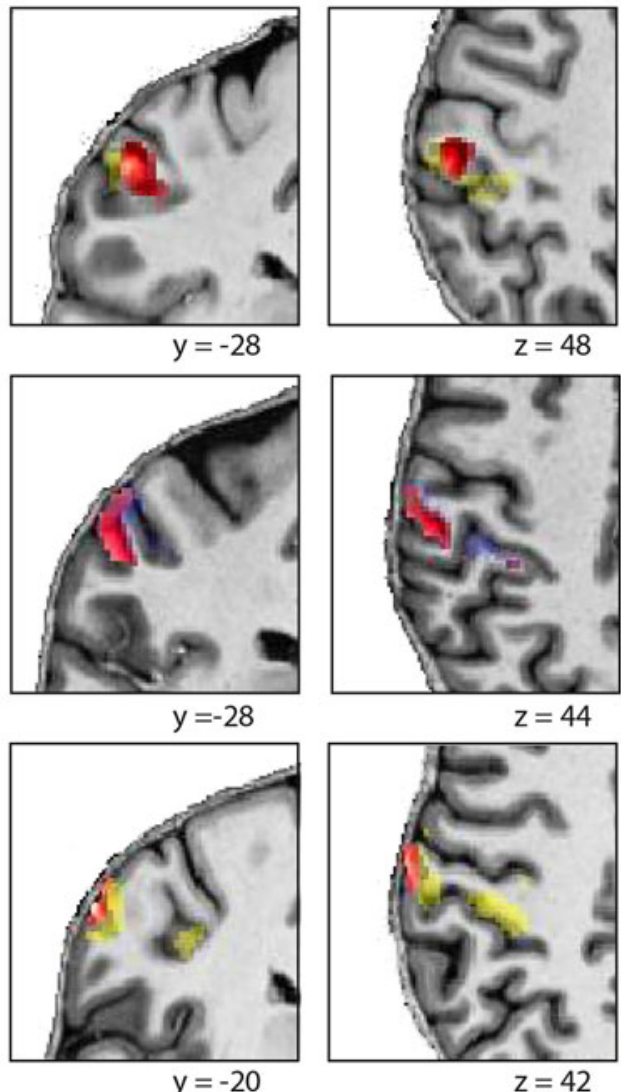

$z=44$

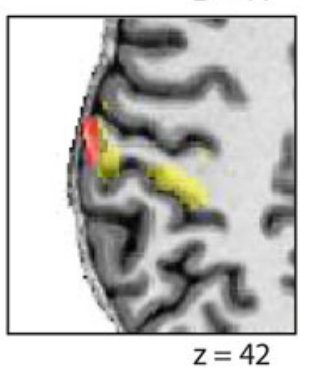

Sub. 4

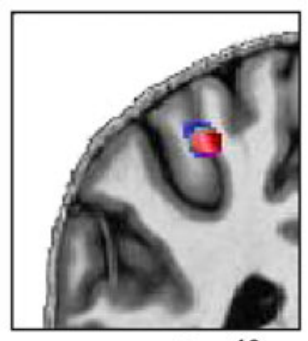

$y=-40$
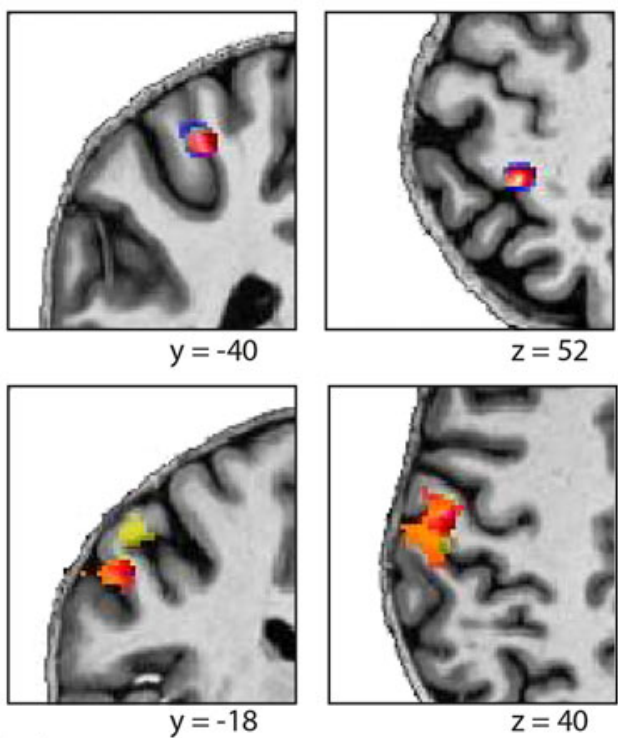

obs. touch IF - no -touch

obs. touch MF - no -touch

SI obs. touch

note that the same axial and coronal slices of the same subjects can here be visually compared; functional data are presented at the individual's normalized T1-anatomical scans; to make both conditions better comparable, a slightly more conservative threshold was chosen for physical touch perception $[p<0.001$ (uncorrected) $]$ than for touch observation $[p<0.005$ (uncorrected)] 
touch did not reveal any significant activity changes in S1. At the individual subject level, $n=8$ participants showed a suppressive interaction effect for self-related observed touch, and $n=4$ participants showed a suppressive interaction effect for other-related observed touch. The mean IR for self-related observed touch was $54.93 \%, \mathrm{SD}=11.35$. The mean IR for observed touch (main effect) was $50.22 \%, \mathrm{SD}=9.35$.

\section{The role of overlapping RFs}

We found that the suppressive interaction effect for physical touch occurred in both overlapping and non-overlapping S1 voxels. More precisely, we found part of the cluster of the suppressive interaction effect for physical touch in overlapping voxels $[k=113 ; t=7.47 ;-54$, $-26,39(x, y, z)$, localized in left area 2, the left inferior parietal cortex, and left area 1] and part of the cluster in non-overlapping voxels $[k=7, t=4.74,-52,-21,38$ ( $x$, $y, z)$, localized in left area 2 , the left inferior parietal cortex, and left area 3b]. Also at the individual subject level, the suppressive interaction effect for physical touch occurred within both overlapping and non-overlapping $\mathrm{S} 1$ voxels in all subjects. As a mean across participants, $79 \%$ of the suppressive interaction voxels were overlapping voxels, whereas $21 \%$ were non-overlapping voxels.

The suppressive interaction effect for observed touch was, on the group level, only observed in non-overlapping $\mathrm{S} 1$ voxels $[k=14 ; t=4.61 ;-56,-24,45(x, y, z)$, localized in left area 2]. When looking at the individual subject level, however, the effect was found both in overlapping and non-overlapping S1 voxels in all participants. Across participants, $60 \%$ of the voxels that showed a suppressive interaction effect were overlapping voxels, and the remaining $40 \%$ were non-overlapping voxels. For selfrelated observed touch, again suppressive interactions at the group level were only found in non-overlapping S1 voxels $[k=14 ; t=4.62 ;-42,-40,62(x, y, z)$, localized in left area $2, k=9 ; t=4.93 ;-49,-36,57(x, y, z)]$. At the single subject level, however, the effect was again found in both overlapping and non-overlapping S1 voxels. Here, $56 \%$ of the voxels that showed suppressive interactions for self-related observed touch were overlapping voxels, and the remaining $44 \%$ were non-overlapping voxels.

\section{Behavioral results}

All participants performed the visual roughness discrimination task (where participants had to distinguish between roughness levels of different paintbrush pairs by sight) with high levels of accuracy [self touch: $93.1 \% \pm 5.4$ (SD), self no-touch: $95.0 \% \pm 7.6$ (SD), other touch: $91.8 \% \pm 7.4$
(SD), other no-touch: $91.7 \% \pm 7.0$ (SD), $N=15]$. The participants maximally missed two trials throughout the entire experiment. The percentage of correct responses in the visual roughness discrimination task was not significantly influenced by hand identity (self, other) or the presence of hand touch (observed touch, no-touch). There was also no significant interaction between these two factors $(p>0.1)$. Also, the finger that was touched (IF, MF, BF) did not influence the percentage of correct responses, neither across conditions nor for the self- and other-related conditions separately $(p>0.05)$. With respect to how individual IRs related to the degree to which roughness levels could be distinguished by sight, there was a significant correlation between the individual IRs when the self was observed and the accuracy to solve the visual roughness discrimination task when self-related touch to the IF and to BF was observed ( $r=0.78$ for IF, and $r=0.76$ for BF self, $p<0.05$, two-tailed, see Online Resource 3). There was no such relation between the individual IRs during observed touch and percentage of accuracy across conditions. Note, however, that the number of subjects whose data were available to calculate this correlation $(n=9)$ was very small, such that this positive relation between individual IRs and behavioral performance has to be replicated and verified by future studies using a greater number of participants.

\section{Discussion}

The present study offers the first detailed characterization of the functional architecture of S1 during touch observation. Our data show that posterior parts of contralateral S1 in particular, but not anterior parts, are activated when touch is observed on video. Activity changes in posterior S1 elicited by touch observation also overlap with those elicited by physical touch perception. Importantly, observing touch to the index finger alone or the middle finger alone offers a similar topographical arrangement of RFs in S1 as those elicited by physically perceiving touch to the same fingers. In addition, index and middle finger RFs show the characteristic dynamic shrinkage when activated concurrently not only during physical but also during visual touch perception. Our study, therefore, provides novel evidence indicating that the functional architecture in posterior $\mathrm{S} 1$ with respect to RF topography and $\mathrm{RF}$ interaction is similar between touch observation and physical touch perception.

Posterior S1 activity during touch observation

In the present study, short video clips were presented to participants, which showed right hands being touched or 
not being touched by paintbrushes. By comparing observed touch conditions to conditions where no touch was observed, we found significant activity increases in left (contralateral) S1 as a main effect. This effect was not only found at the group level but also in almost all individual participants. Significant activity changes in right (ipsilateral) S1 were only found when the group level statistics were analyzed at uncorrected thresholds. The results of the present study show that observing touch to fingers of a right hand, therefore, clearly evokes activity increases in left posterior $\mathrm{S} 1$.

The finding that touch observation can elicit activity increases in $\mathrm{S} 1$ is in accordance with a growing body of evidence suggesting the independence of S1 activity from direct somatosensory input (Chen et al. 2003; Yoo et al. 2003; Driver and Noesselt 2008; Meehan et al. 2009; Wood et al. 2010). Specifically touch observation has, in a number of fMRI studies, been shown to trigger profound activity increases in S1 (Blakemore et al. 2005; Ebisch et al. 2008; Kuehn et al. 2012; Schaefer et al. 2005, 2006, 2009). A previous study also indicated that in particular posterior rather than anterior contralateral S1 responds to the observation of tactile events (Kuehn et al. 2012). This appears to contrast findings reported in an fMRI study by Schaefer et al. (2009). Participants in that study also observed short video sequences where hands were either touched or not touched by paintbrushes. Whereas, in accordance with the present results and those previously reported, touch observation induced activity increases in posterior contralateral $\mathrm{S} 1$, the authors also reported responsivity of anterior $\mathrm{S} 1$, specifically when participants looked at a hand presented in a first-person viewing perspective. A recent study reinvestigating this topic using $7 \mathrm{~T}$ fMRI (Kuehn et al. 2012) yielded divergent findings. In that study, anterior S1 did not show significant activity changes, neither as a main effect, nor when first-person and third-person viewing perspectives were directly compared. Kuehn et al. (2012) argued that the lower spatial resolution of the data used by Schaefer et al. (2009), in terms of voxel size and smoothing, may have accounted for the divergent findings. Although an involvement of anterior $\mathrm{S} 1$ cannot be excluded, it seems that the major responsivity of S1 during touch observation stems from its posterior parts. This is also in accordance with the recently formulated hypothesis that posterior S1 in particular is open to social influences, for example during action observation (Keysers et al. 2010).

The high connectivity between posterior S1 and visual input areas in the parietal cortex, some of which are known to contain bimodal visuo-tactile neurons (Duhamel et al. 1998; Ishida et al. 2010; Lewis and Van Essen 2000; Maunsell and van Essen 1983; Pons and Kaas 1986; Rozzi et al. 2006) and to show bimodal activation pattern in humans (Sereno and Huang 2006), can serve to explain this greater influence of vision on activity changes in the posterior rather than the anterior part of S1. Anterior S1 is more strongly connected to the thalamus than posterior S1 (Kaas 2008; Nelson and Kaas 1981); therefore, we assume that the thalamus did not strongly contribute to the S1 activity observed in the present study. A dichotomic division of S1 into posterior S1, showing pronounced reactivity to visual input (e.g., during observed touch) and anterior $\mathrm{S} 1$, which may still be regarded as a unisensory brain area mainly driven by bottom-up somatosensory input, has been suggested previously (Keysers et al. 2010; Kuehn et al. 2012), and is supported by our results.

Another important question is whether the activity changes in posterior $\mathrm{S} 1$ found in our study were triggered by touch observation, or resulted from preparatory motor responses or mental imaginary of action. Given the role of area 2 in proprioception (Hsiao and Bensmaia 2008), and the involvement of $\mathrm{S} 1$ in motor preparation (Kawashima et al. 1994), such an explanation cannot a priori be excluded. However, for the present findings, this explanation is highly unlikely. Activity changes in S1 were strongly lateralized to left S1 (contralateral to the observed touch events), whereas right $\mathrm{S} 1$ (contralateral to the motor response) showed only sub-threshold activity. In addition, we did not find any activity increases in left or right M1 during touch observation, which would be expected if one assumed an involvement of motor preparation (Kawashima et al. 1994) or motor imaginary (Dushanova and Donoghue 2010). We are, therefore, confident that the S1 activity reported in the present study is due to touch observation rather than preparatory motor activity or motor imaginary.

\section{Topography of S1 activity during touch observation}

In order to describe the functional architecture of posterior S1 during touch observation, we first looked at whether S1 activity during observed and physically perceived touch showed a regional overlap. Any overlap would indicate a resonance response (Hogeveen and Obhi 2012; Landmann et al. 2011; Virji-Babul et al. 2012) within S1 between physically perceived and observed touch. Such resonance responses have often been described for the motor system (Buccino et al. 2001; Mukamel et al. 2010; see Caspers et al. 2010 and Gazzola and Keysers 2009 for an overview), the insula (Corradi-Dell' Acqua et al. 2011; Singer et al. 2004; see Bernhardt and Singer 2012 and Lamm et al. 2011 for an overview), S2 (Keysers et al. 2004), and also for S1 (Blakemore et al. 2005; Ebisch et al. 2008; Schaefer et al. 2009). However, so far, they have not been characterized with such a high sensitivity and high spatial specificity as offered by the design of the present study. Whereas previous studies indicated spatial specificity of S1 activity when touch to different body areas, such as the face and neck, was 
observed (Blakemore et al. 2005), or showed that observing hand touch elicited specific activity increases in the hand area of S1 (Kuehn et al. 2012; Schaefer et al. 2009), the present study indicates a spatially specific resonance response at the level of the single finger. More precisely, observing touch to the index finger overlapped with activity changes during physically experiencing touching of the index finger, and observing touch to the middle finger overlapped with activity changes during physically experiencing touching of the middle finger. Interestingly, whereas this resonance response seemed relatively specific for the index finger (i.e., S1 responses to observing touch to the index finger significantly overlapped with those to physical touching of the index finger, but did not significantly overlap with those to physical touching of the middle finger), this specificity was not present for observing touch to the middle finger. Here, the same significance level was reached irrespectively of whether the contrast of observing touch to the middle finger was masked with physical touching of the index finger or physical touching of the middle finger. In addition, the responsivity of S1 was generally larger when touching to the index finger was observed compared with when touching to the middle finger was observed. These results indicate that observing touch to the index finger leads to higher and spatially more specific responses in S1 compared to observing touch to the middle finger. These results could be explained by the generally enhanced use of the index finger compared to the middle finger, for example during the so called precision grip (Napier 1956). In studies on the motor system, greater experience in a certain motor behavior has been shown to lead to increased responses in the motor system not only during action performance (Karni et al. 1995, 1998) but also during action observation (Calvo-Merino et al. 2005; Cross et al. 2006). In addition, a more precise response of the action observation network has also been assumed for participants that have more experience with the observed actions (Cross et al. 2012; Diersch et al. 2012). One may therefore argue that the results of the present study indicate a similar relation in the somatosensory system. Increased tactile experience of a certain body area, such as the index finger, that has been shown to relate to increased $\mathrm{S} 1$ activity (Braun et al. 2000; Pleger et al. 2003) and better discrimination abilities (Braun et al. 2000; Ragert et al. 2003; Schweizer et al. 2001) during physical touch perception, may also cause a stronger and more precise representation during touch observation.

It is important to note, however, that the present data do now allow a direct comparison between touch observation and physical touch perception. Whereas in the observed touch videos, different paintbrushes were used for tactile stimulation, and a roughness task had to be solved, in the physical touch experiment, tactile stimulation was applied passively, and the same paintbrushes were used for tactile stimulation. The experimental set-ups therefore differ, and do not allow direct comparison of S1 RFs as evoked by visual and physical touch perception. Future studies should use completely analogue designs with respect to stimulus characteristics and attention requirements in order to compare the overlap between RFs in both conditions more precisely. Only such a design would finally allow conclusions to be drawn about the specificity of the activity overlap between physical touch perception and touch observation.

A second main aspect that characterizes S1 topography during touch observation is the topographical arrangement of the evoked activity changes. Our results show that activity changes in $\mathrm{S} 1$ during touch observation to the index finger were partly distinct, and located more lateral, more anterior, and more inferior than activity changes in S1 during touch observation to the middle finger. This topographical alignment of index and middle finger RFs follows exactly the same pattern as has classically been described for physical touch (Nelson and Chen 2008), and as has also been found in the present study. This indicates a surprisingly precise representation of observed touch events in S1, and assumes a precision down to the level of the single finger. Should further studies manifest this finding, this would offer another parallel to the action system. Also, observing motor movements of specific body parts has been shown to elicit somatotopically precise representations in the premotor cortex (Buccino et al. 2004; Wheaton et al. 2004); the present study assumes a similarly spatially specific and precise representation of observed human touch.

Taken together, our results indicate that observing touch to single fingers does not simply activate the hand area in $\mathrm{S} 1$, but activates parts in $\mathrm{S} 1$ that are topographically precise. The spatial arrangement of S1 activity seems therefore highly similar during physically perceived and observed touch, which leads to the suggestion that not only action events but also tactile events can be shared between the observed person and the observer (Bufalari et al. 2007).

\section{Suppressive interactions during touch observation}

In the present study, the functional architecture in S1 was additionally characterized by looking at suppressive interactions between adjacently activated cortical RFs. Suppressive interactions in S1 have often been characterized by measuring the relative shrinkage of index and middle finger RFs when both are activated simultaneously, compared to when they are activated alone (Gandevia et al. 1983; Ruben et al. 2006). Using this approach in the present study, we found suppressive interactions mainly in posterior parts of contralateral S1, slightly extending to anterior S1. This confirms previous studies that found greater suppressive interactions during touch perception in 
posterior contralateral S1 (Friedman et al. 2008; Ruben et al. 2006; Sur 1980; Sripati et al. 2006), which may indicate an increasing convergence of somatosensory input from anterior to posterior sites of S1 (Ruben et al. 2006). Also the mean interaction ratio found in the present study (38\%) was similar to that which has been described previously (Biermann et al. 1998; Gandevia et al. 1983; Ruben et al. 2006). These comparable results between the present and previous attempts to characterize suppressive interactions in S1 confirm that the present approach was, in principle, suitable to characterize this phenomenon.

This is important given that analogue contrasts were used to characterize suppressive interactions during touch observation. This characterization was attempted for the first time in the present study. Here, we looked at whether suppressive interactions in S1 would similarly occur when touch to two fingers, compared to two single fingers separately, was not physically experienced but merely observed. Our data indicate that suppressive interactions in S1 may also occur during touch observation. More precisely, we found that observing touch to two fingers elicited decreased activity levels in S1 compared to observing touch to two single fingers separately, an effect that was specific for the areas in S1 where observed touch to single fingers separately elicited effects. Spatially, the effect was restricted to contralateral posterior $\mathrm{S} 1$, which was expected given that touch observation particularly activated posterior parts of contralateral S1. Importantly, suppressive interactions were also found in voxels that did not overlap between index and middle finger RFs, making vascular ceiling or saturation effects an unlikely explanation for the observed effects (Beauchamp et al. 2004; Gardner and Costanzo 1980).

It is important to note, however, that while the principle way of characterizing suppressive interactions during visual and physical touch perception in the present study was similar, the results of these two analyses should not be compared directly. During physical touch perception, participants lay in the scanner with their eyes closed, while they actively solved a roughness discrimination task during touch observation. Because previous research has evidenced an influence of attention on suppressive interactions in S1 (Braun et al. 2002), the suppressive interaction effect in both conditions is not directly comparable because attentional demands varied between both experiments. Secondly, there was a difference in control conditions. During touch observation, S1 activity changes were compared to a control condition (i.e., where participants saw hands which were not being touched), whereas in the physical touch condition, no such control condition was present (i.e., physical touch perception was compared to a rest condition). Given that merely looking at hands may influence S1 activity (Fiorio and Haggard 2005; Longo et al. 2011) and the degree of suppressive interactions in S1
(Cardini et al. 2011), one should avoid comparing the degree of suppressive interactions between physical and visual touch perception in the present study directly.

The indicated existence of suppressive interactions in S1 during touch observation can be embedded into the results from recent studies that assign $\mathrm{S} 1$ a specific and highly flexible role during touch and action observation (Avenanti et al. 2005; Bolognini et al. 2011; Bufalari et al. 2007; Caspers et al. 2010; Keysers et al. 2010; Meyer et al. 2011). For instance, using multivariate pattern analysis, it has been shown that activity patterns in S1 are separable when haptic exploration of different everyday objects is observed (Meyer et al. 2011). Such variable and spatially specific activity changes in $\mathrm{S} 1$ could be regarded as an indication of the existence of inhibitory regulatory mechanisms that modulate S1 activity during touch observation. More direct evidence that vision can influence the degree of suppressive interactions in S1 during physical touch perception is offered by studies investigating somatosensory evoked potentials (SEPs) in different viewing conditions. Here, it has been assumed that looking at a body while receiving tactile stimulation increases suppressive interactions in S1 (Cardini et al. 2011; Gillmeister et al. 2010), which has been related to RF sharpening in this condition (Cardini et al. 2011; Haggard et al. 2007). Whereas the results of the present study are, therefore, in accordance with previous investigations, they are novel because they target the mechanism of suppressive interactions during touch observation for the first time directly.

The relation between suppressive interactions and behavioral performance

During physical touch perception, suppressive interactions are assumed to positively relate to perceived stimulus contrast (Braun et al. 2002; Cardini et al. 2011; Puts et al. 2011). We therefore hypothesized that, if suppressive interactions during physical and observed touch share a mechanistic basis, such a relation to the ability to discriminate tactile stimulus features should also occur during touch observation. This analysis, however, was hampered by the very small sample size available to calculate this correlation $(n=9)$. However, when looking at the correlation, the degree of suppressive interactions during observed touch related positively to performance levels to discriminate roughness levels of paintbrushes by sight. Although this relation clearly needs further exploration in future studies, it indicates a powerful message: the degree of suppressive interactions in S1 during observed touch may determine the precision with which observed tactile events can be decoded by the observer. This is particularly interesting because, so far, signal decreases in S1 during touch observation are mostly assumed to indicate a lower resonance response, and are, thus, interpreted as evidence 
for lower degrees of inner simulation (Blakemore et al. 2005; Ebisch et al. 2008; Kuehn et al. 2012). Given the results of the present study, this view may be rather onedimensional. Signal decreases, at least when they can clearly be assigned to the occurrence of suppressive interactions, may indicate a more precise and less noisy, rather than a weaker, stimulus representation. This similarly holds for action observation. A recent study showed that the BOLD response in the action observation network, which is classically assumed to increase during observed actions that are more familiar (Buccino et al. 2004; CalvoMerino et al. 2005; Cross et al. 2006), does not increase when more familiar actions compared to less familiar actions are observed (Cross et al. 2011). Given our framework, one may speculate that the decreases in the BOLD signal indicate a more precise representation of the observed familiar movements. Future studies should, therefore, take decreases of the BOLD signal into account when investigating the role of S1, or other brain areas, in the realm of social cognition.

\section{Conclusions}

Taken together, the results from our study provide strong evidence that posterior contralateral S1 is active during touch observation, and that these activity changes overlap with those elicited by physical touch experience. In addition, our results indicate that touch observation to single fingers elicits partly distinct and topographically precise single finger representations in $\mathrm{S} 1$, which show similar dynamic interactions as they do during physical touch perception. Although this study only provides a first step to understanding the functional architecture of $\mathrm{S} 1$ in a social context, it critically emphasizes the importance of taking fine-grained architectonic details into account when describing the role of $\mathrm{S} 1$ in social cognition.

\begin{abstract}
Acknowledgments We gratefully acknowledge the funding of this work by the International Max Planck Research School on Neuroscience of Communication: Function, Structure, and Plasticity. This work was also supported by the Max Planck Society. We additionally thank Domenica Wilfing, Elisabeth Wladimirow, Clara Kühn, and Manja Luckner for their help during data acquisition.
\end{abstract}

Open Access This article is distributed under the terms of the Creative Commons Attribution License which permits any use, distribution, and reproduction in any medium, provided the original author(s) and the source are credited.

\section{References}

Ashburner J, Friston KJ (2005) Unified segmentation. Neuroimage 26(3):839-851
Avenanti A, Bueti D, Galati G, Aglioti SM (2005) Transcranial magnetic stimulation highlights the sensorimotor side of empathy for pain. Nat Neurosci 8(7):955-960

Avenanti A, Bolognini N, Maravita A, Aglioti SM (2007) Somatic and motor components of action simulation. Curr Biol 17(24): 2129-2135

Bandettini PA (2009) What's new in neuroimaging methods? Ann N Y Acad Sci 1156:260-293

Beauchamp MS, Argall BD, Bodurka J, Duyn JH, Martin A (2004) Unraveling multisensory integration: patchy organization within human STS multisensory cortex. Nat Neurosci 7(11):1190-1192

Beauchamp MS, Laconte S, Yasar N (2009) Distributed representation of single touches in somatosensory and visual cortex. Hum Brain Mapp 30(10):3163-3171

Bernhardt B, Singer T (2012) The neural basis of empathy. Annu Rev Neurosci 35:1-23

Biermann K, Schmitz F, Witte OW, Konczak J, Freund HJ, Schnitzler A (1998) Interaction of finger representation in the human first somatosensory cortex: a neuromagnetic study. Neurosci Lett 251(1):13-16

Blakemore SJ, Bristow D, Bird G, Frith C, Ward J (2005) Somatosensory activations during the observation of touch and a case of vision-touch synaesthesia. Brain 128(Pt 7):1571-1583

Blankenburg F, Ruben J, Meyer R, Schwiemann J, Villringer A (2003) Evidence for a rostral-to-caudal somatotopic organization in human primary somatosensory cortex with mirror-reversal in areas $3 b$ and 1. Cereb Cortex 13(9):987-993

Bolognini N, Rossetti A, Maravita A, Miniussi C (2011) Seeing touch in the somatosensory cortex: a TMS study of the visual perception of touch. Hum Brain Mapp 32(12):2104-2114

Braun C, Schweizer R, Elbert T, Birbaumer N, Taub E (2000) Differential activation in somatosensory cortex for different discrimination tasks. J Neurosci 20(1):446-450

Braun C, Haug M, Wiech K, Birbaumer N, Elbert T, Roberts LE (2002) Functional organization of primary somatosensory cortex depends on the focus of attention. Neuroimage 17(3):1451-1458

Buccino G, Binkofski F, Fink G, Fadiga L, Fogassi L, Gallese V, Seitz R, Zilles K, Rizzolatti G, Freund H (2001) Action observation activates premotor and parietal areas in a somatotopic manner: an fMRI study. Eur J Neurosci 13(2):400-404

Buccino G, Binkofski F, Riggio L (2004) The mirror neuron system and action recognition. Brain Lang 89(2):370-376

Bufalari I, Aprile T, Avenanti A, Di Russo F, Aglioti SM (2007) Empathy for pain and touch in the human somatosensory cortex. Cereb Cortex 17(11):2553-2561

Calvo-Merino B, Glaser DE, Grezes J, Passingham RE, Haggard P (2005) Action observation and acquired motor skills: an FMRI study with expert dancers. Cereb Cortex 15(8):1243-1249

Cardini F, Longo MR, Haggard P (2011) Vision of the body modulates somatosensory intracortical inhibition. Cereb Cortex 21(9):2014-2022

Caspers S, Zilles K, Laird AR, Eickhoff SB (2010) ALE metaanalysis of action observation and imitation in the human brain. Neuroimage 50(3):1148-1167

Chen LM, Friedman RM, Roe AW (2003) Optical imaging of a tactile illusion in area $3 \mathrm{~b}$ of the primary somatosensory cortex. Science 302(5646):881-885

Chen LM, Turner GH, Friedman RM, Zhang N, Gore JC, Roe AW, Avison MJ (2007) High-resolution maps of real and illusory tactile activation in primary somatosensory cortex in individual monkeys with functional magnetic resonance imaging and optical imaging. J Neurosci 27(34):9181-9191

Corradi-Dell'Acqua C, Hofstetter C, Vuilleumier P (2011) Felt and seen pain evoke the same local patterns of cortical activity in insular and cingulate cortex. J Neurosci 31(49):17996-18006 
Cross ES, Hamilton AF, Grafton ST (2006) Building a motor simulation de novo: observation of dance by dancers. Neuroimage 31(3): 1257-1267

Cross ES, Stadler W, Parkinson J, Schütz-Bosbach S, Prinz W (2011). The influence of visual training on predicting complex action sequences. Hum Brain Map. doi:10.1002/hbm.21450

Cross ES, Liepelt R, de Hamilton C, Parkinson J, Ramsey R, Stadler W, Prinz W (2012) Robotic movement preferentially engages the action observation network. Hum Brain Mapp 33(9):2238-2254

DiCarlo JJ, Johnson KO (2002) Receptive field structure in cortical area $3 b$ of the alert monkey. Behav Brain Res 135(1-2):167-178

DiCarlo JJ, Johnson KO, Hsiao SS (1998) Structure of receptive fields in area $3 \mathrm{~b}$ of primary somatosensory cortex in the alert monkey. J Neurosci 18(7):2626-2645

Diersch N, Cross E, Stadler W, Rieger M, Schütz-Bosbach S (2012) Action prediction in younger versus older adults: Neural correlates of motor familiarity

Dionne JK, Meehan SK, Legon W, Staines WR (2010) Crossmodal influences in somatosensory cortex: interaction of vision and touch. Hum Brain Mapp 31(1):14-25

Driver J, Noesselt T (2008) Multisensory interplay reveals crossmodal influences on 'sensory-specific' brain regions, neural responses, and judgments. Neuron 57(1):11-23

Duhamel JR, Colby CL, Goldberg ME (1998) Ventral intraparietal area of the macaque: congruent visual and somatic response properties. J Neurophysiol 79(1):126-136

Duncan RO, Boynton GM (2007) Tactile hyperacuity thresholds correlate with finger maps in primary somatosensory cortex (S1). Cereb Cortex 17(12):2878-2891

Dushanova J, Donoghue J (2010) Neurons in primary motor cortex engaged during action observation. Eur J Neurosci 31(2):386-398

Dykes RW (1983) Parallel processing of somatosensory information: a theory. Brain Res 287(1):47-115

Ebisch SJ, Perrucci MG, Ferretti A, Del Gratta C, Romani GL, Gallese V (2008) The sense of touch: embodied simulation in a visuotactile mirroring mechanism for observed animate or inanimate touch. J Cogn Neurosci 20(9):1611-1623

Eickhoff SB, Stephan KE, Mohlberg H, Grefkes C, Fink GR, Amunts $\mathrm{K}$, Zilles K (2005) A new SPM toolbox for combining probabilistic cytoarchitectonic maps and functional imaging data. Neuroimage 25(4):1325-1335

Eickhoff SB, Heim S, Zilles K, Amunts K (2006) Testing anatomically specified hypotheses in functional imaging using cytoarchitectonic maps. Neuroimage 32(2):570-582

Eickhoff SB, Paus T, Caspers S, Grosbras MH, Evans AC, Zilles K, Amunts K (2007) Assignment of functional activations to probabilistic cytoarchitectonic areas revisited. Neuroimage 36(3):511-521

Eimer M, Cockburn D, Smedley B, Driver J (2001) Cross-modal links in endogenous spatial attention are mediated by common external locations: evidence from event-related brain potentials. Exp Brain Res 139(4):398-411

Fabbri-Destro M, Rizzolatti G (2008) Mirror neurons and mirror systems in monkeys and humans. Physiology 23:171-179

Falkner AL, Krishna BS, Goldberg ME (2010) Surround suppression sharpens the priority map in the lateral intraparietal area. J Neurosci 30(38):12787-12797

Fiorio M, Haggard P (2005) Viewing the body prepares the brain for touch: effects of TMS over somatosensory cortex. Eur J Neurosci 22(3):773-777

Fitzgibbon BM, Giummarra MJ, Georgiou-Karistianis N, Enticott PG, Bradshaw JL (2010) Shared pain: from empathy to synaesthesia. Neurosci Biobehav Rev 34(4):500-512

Friedman RM, Chen LM, Roe AW (2008) Responses of areas 3b and 1 in anesthetized squirrel monkeys to single- and dual-site stimulation of the digits. J Neurophysiol 100(6):3185-3196
Gandevia SC, Burke D, McKeon BB (1983) Convergence in the somatosensory pathway between cutaneous afferents from the index and middle fingers in man. Exp Brain Res 50(2-3): $415-425$

Gardner EP, Costanzo RM (1980) Spatial integration of multiplepoint stimuli in primary somatosensory cortical receptive fields of alert monkeys. J Neurophysiol 43(2):420-443

Gardner E, Kass JH (2008) The senses, vol 6, Somatosensation, 1st edn. Elsevier, Canada

Gati JS, Menon RS, Ugurbil K, Rutt BK (1997) Experimental determination of the BOLD field strength dependence in vessels and tissue. Magn Reson Med 38(2):296-302

Gazzola V, Keysers C (2009) The observation and execution of actions share motor and somatosensory voxels in all tested subjects: single-subject analyses of unsmoothed fMRI data. Cereb Cortex 19(6):1239-1255

Geyer S, Schleicher A, Zilles K (1999) Areas 3a, 3b, and 1 of human primary somatosensory cortex. Neuroimage 10(1):63-83

Geyer S, Schormann T, Mohlberg H, Zilles K (2000) Areas 3a, 3b, and 1 of human primary somatosensory cortex. Part 2. Spatial normalization to standard anatomical space. Neuroimage 11(6 Pt 1):684-696

Gillmeister H, Sambo CF, Forster B (2010) Which finger? Early effects of attentional selection within the hand are absent when the hand is viewed. Eur J Neurosci 31(10):1874-1881

Grefkes C, Geyer S, Schormann T, Roland P, Zilles K (2001) Human somatosensory area 2: observer-independent cytoarchitectonic mapping, interindividual variability, and population map. Neuroimage 14(3):617-631

Griswold MA, Jakob PM, Heidemann RM, Nittka M, Jellus V, Wang J, Kiefer B, Haase A (2002) Generalized autocalibrating partially parallel acquisitions (GRAPPA). Magn Reson Med 47(6):1202-1210

Haavik Taylor H, Murphy BA (2007) Altered cortical integration of dual somatosensory input following the cessation of a $20 \mathrm{~min}$ period of repetitive muscle activity. Exp Brain Res 178(4):488-498

Haggard P, Christakou A, Serino A (2007) Viewing the body modulates tactile receptive fields. Exp Brain Res 180(1):187-193

Heidemann RM, Ivanov D, Trampel R, Fasano F, Meyer H, Pfeuffer J, Turner R (2012) Isotropic submillimeter fMRI in the human brain at $7 \mathrm{~T}$ : combining reduced field-of-view imaging and partially parallel acquisitions. Magn Res Med 68(5):1506-1516

Hoechstetter K, Rupp A, Stancak A, Meinck HM, Stippich C, Berg P, Scherg M (2001) Interaction of tactile input in the human primary and secondary somatosensory cortex - a magnetoencephalographic study. Neuroimage 14(3):759-767

Hogeveen J, Obhi SS (2012) Social interaction enhances motor resonance for observed human actions. J Neurosci 32(17):5984-5989

Hsiao SS, Bensmaia S (2008) Coding of object shape and texture. In: Gardner E, Kaas J (eds) The senses, vol 6, somatosensation, 1st edn. Elsevier, Canada, pp 55-66

Hsiao SS, O'Shaughnessy DM, Johnson KO (1993) Effects of selective attention on spatial form processing in monkey primary and secondary somatosensory cortex. J Neurophysiol 70(1):444-447

Hsieh CL, Shima F, Tobimatsu S, Sun SJ, Kato M (1995) The interaction of the somatosensory evoked potentials to simultaneous finger stimuli in the human central nervous system. A study using direct recordings. Electroencephalogr Clin Neurophysiol 96(2):135-142

Ishibashi H, Tobimatsu S, Shigeto H, Morioka T, Yamamoto T, Fukui M (2000) Differential interaction of somatosensory inputs in the human primary sensory cortex: a magnetoencephalographic study. Clin Neurophysiol Off $\mathrm{J}$ Int Fed Clin Neurophysiol 111(6):1095-1102

Ishida H, Nakajima K, Inase M, Murata A (2010) Shared mapping of own and others' bodies in visuotactile bimodal area of monkey parietal cortex. J Cogn Neurosci 22(1):83-96 
Jones EG (1993) GABAergic neurons and their role in cortical plasticity in primates. Cereb Cortex 3(5):361-372

Kaas JH (2008) The somatosensory thalamus and associated pathways. In: Gardner E, Kass JH (eds) The senses, vol 6, Somatosensation, 1st edn. Elsevier, Canada, pp 117-142

Kaas JH, Jain N, Qi HX (2002) The organization of the somatosensory system in primates. In: Nelson RJ (ed) The somatosensory system: deciphering the brain's own body image. CRC Press, Boca Raton, pp 1-26

Karni A, Meyer G, Jezzard P, Adams MM, Turner R, Ungerleider LG (1995) Functional MRI evidence for adult motor cortex plasticity during motor skill learning. Nature 377(6545):155-158

Karni A, Meyer G, Rey-Hipolito C, Jezzard P, Adams MM, Turner R, Ungerleider LG (1998) The acquisition of skilled motor performance: fast and slow experience-driven changes in primary motor cortex. Proc Natl Acad Sci USA 95(3):861-868

Kawashima R, Roland PE, O'Sullivan BT (1994) Fields in human motor areas involved in preparation for reaching, actual reaching, and visuomotor learning: a positron emission tomography study. J Neurosci 14(6):3462-3474

Kayser C (2010) The multisensory nature of unisensory cortices: a puzzle continued. Neuron 67(2):178-180

Keysers C, Wicker B, Gazzola V, Anton JL, Fogassi L, Gallese V (2004) A touching sight: SII/PV activation during the observation and experience of touch. Neuron 42(2):335-346

Keysers C, Kaas JH, Gazzola V (2010) Somatosensation in social perception. Nat Rev Neurosci 11(6):417-428

Krause T, Kurth R, Ruben J, Schwiemann J, Villringer K, Deuchert M, Moosmann M, Brandt S, Wolf K, Curio G, Villringer A (2001) Representational overlap of adjacent fingers in multiple areas of human primary somatosensory cortex depends on electrical stimulus intensity: an fMRI study. Brain Res 899(1-2):36-46

Kuehn E, Trampel R, Mueller K, Turner R, Schutz-Bosbach S (2012) Judging roughness by sight-A 7-tesla fMRI study on responsivity of the primary somatosensory cortex during observed touch of self and others. Hum Brain Mapp. doi:10.1002/hbm.22031

Lamm C, Decety J, Singer T (2011) Meta-analytic evidence for common and distinct neural networks associated with directly experienced pain and empathy for pain. Neuroimage 54(3):2492-2502

Landmann C, Landi SM, Grafton ST, Della-Maggiore V (2011) FMRI supports the sensorimotor theory of motor resonance. PLoS One 6(11):e26859. doi:10.1371/journal.pone.0026859

Lewis JW, Van Essen DC (2000) Corticocortical connections of visual, sensorimotor, and multimodal processing areas in the parietal lobe of the macaque monkey. J Comp Neurol 428(1):112-137

Longo MR, Haggard P (2010) An implicit body representation underlying human position sense. PNAS 107(26):11727-11732

Longo MR, Pernigo S, Haggard P (2011) Vision of the body modulates processing in primary somatosensory cortex. Neurosci Lett 489(3):159-163

Macaluso E, Frith CD, Driver J (2002) Directing attention to locations and to sensory modalities: multiple levels of selective processing revealed with PET. Cereb Cortex 12(4):357-368

Marques JP, Kober T, Krueger G, van der Zwaag W, Van de Moortele PF, Gruetter R (2010) MP2RAGE, a self bias-field corrected sequence for improved segmentation and T1-mapping at high field. Neuroimage 49(2):1271-1281

Maunsell JH, van Essen DC (1983) The connections of the middle temporal visual area (MT) and their relationship to a cortical hierarchy in the macaque monkey. J Neurosci 3(12):2563-2586

Meehan SK, Legon W, Staines WR (2009) Spatiotemporal properties modulate intermodal influences on early somatosensory processing during sensory-guided movement. Clinical Neurophysiol Off J Int Fed Clin Neurophysiol 120(7):1371-1380
Meyer K, Kaplan JT, Essex R, Damasio H, Damasio A (2011) Seeing touch is correlated with content-specific activity in primary somatosensory cortex. Cereb Cortex 21(9):2113-2121

Moore CI, Nelson SB, Sur M (1999) Dynamics of neuronal processing in rat somatosensory cortex. Trends Neurosci 22(11):513-520

Moore CI, Stern CE, Corkin S, Fischl B, Gray AC, Rosen BR, Dale AM (2000) Segregation of somatosensory activation in the human rolandic cortex using fMRI. J Neurophysiol 84(1):558-569

Mukamel R, Ekstrom AD, Kaplan J, Iacoboni M, Fried I (2010) Single-neuron responses in humans during execution and observation of actions. Curr Biol 20(8):750-756

Naka D, Kakigi R, Koyama S, Xiang J, Suzuki H (1998) Effects of tactile interference stimulation on somatosensory evoked magnetic fields following tibial nerve stimulation. Electroencephalogr Clin Neurophysiol 109(2):168-177

Napier JR (1956) The prehensile movements of the human hand. J Bone Joint Surg 38(B):902

Nelson AJ, Chen R (2008) Digit somatotopy within cortical areas of the postcentral gyrus in humans. Cereb Cortex 18(10):2341-2351

Nelson RJ, Kaas JH (1981) Connections of the ventroposterior nucleus of the thalamus with the body surface representations in cortical areas $3 \mathrm{~b}$ and 1 of the cynomolgus macaque (Macaca fascicularis). J Comp Neurol 199(1):29-64

Oldfield RC (1971) The assessment and analysis of handedness: the Edinburgh inventory. Neuropsychologia 9:17

Pleger B, Foerster AF, Ragert P, Dinse HR, Schwenkreis P, Malin JP, Nicolas V, Tegenthoff M (2003) Functional imaging of perceptual learning in human primary and secondary somatosensory cortex. Neuron 40(3):643-653

Pleger B, Blankenburg F, Ruff CC, Driver J, Dolan RJ (2008) Reward facilitates tactile judgments and modulates hemodynamic responses in human primary somatosensory cortex. J Neurosci 28(33):8161-8168

Pons TP, Kaas JH (1986) Corticocortical connections of area 2 of somatosensory cortex in macaque monkeys: a correlative anatomical and electrophysiological study. J Comp Neurol 248(3):313-335

Puts NA, Edden RA, Evans CJ, McGlone F, McGonigle DJ (2011) Regionally specific human GABA concentration correlates with tactile discrimination thresholds. J Neurosci 31(46):16556-16560

Ragert P, Dinse HR, Pleger B, Wilimzig C, Frombach E, Schwenkreis $\mathrm{P}$, Tegenthoff $\mathrm{M}$ (2003) Combination of $5 \mathrm{~Hz}$ repetitive transcranial magnetic stimulation (rTMS) and tactile coactivation boosts tactile discrimination in humans. Neurosci Lett 348 (2):105-108

Rossetti A, Miniussi C, Maravita A, Bolognini N (2012) Visual perception of bodily interactions in the primary somatosensory cortex. Eur J Neurosci 36(3):2317-2323

Rozzi S, Calzavara R, Belmalih A, Borra E, Gregoriou GG, Matelli M, Luppino G (2006) Cortical connections of the inferior parietal cortical convexity of the macaque monkey. Cereb Cortex 16(10):1389-1417

Ruben J, Krause T, Taskin B, Blankenburg F, Moosmann M, Villringer A (2006) Sub-area-specific Suppressive Interaction in the BOLD responses to simultaneous finger stimulation in human primary somatosensory cortex: evidence for increasing rostral-to-caudal convergence. Cereb Cortex 16(6):819-826

Sanchez-Panchuelo RM, Francis S, Bowtell R, Schluppeck D (2010) Mapping human somatosensory cortex in individual subjects with 7 T functional MRI. J Neurophysiol 103(5):2544-2556

Sastre-Janer FA, Regis J, Belin P, Mangin JF, Dormont D, Masure MC, Remy P, Frouin V, Samson Y (1998) Three-dimensional reconstruction of the human central sulcus reveals a morphological correlate of the hand area. Cereb Cortex 8(7):641-647 
Schaefer M, Heinze HJ, Rotte M (2005) Seeing the hand being touched modulates the primary somatosensory cortex. NeuroReport 16(10):1101-1105

Schaefer M, Flor H, Heinze HJ, Rotte M (2006) Dynamic modulation of the primary somatosensory cortex during seeing and feeling a touched hand. Neuroimage 29(2):587-592

Schaefer M, Xu B, Flor H, Cohen LG (2009) Effects of different viewing perspectives on somatosensory activations during observation of touch. Hum Brain Mapp 30(9):2722-2730

Schaefer M, Heinze HJ, Rotte M (2012) Embodied empathy for tactile events: interindividual differences and vicarious somatosensory responses during touch observation. Neuroimage 60(2):952-957

Schweizer R, Braun C, Fromm C, Wilms A, Birbaumer N (2001) The distribution of mislocalizations across fingers demonstrates training-induced neuroplastic changes in somatosensory cortex. Exp Brain Res 139(4):435-442

Scouten A, Papademetris X, Constable RT (2006) Spatial resolution, signal-to-noise ratio, and smoothing in multi-subject functional MRI studies. Neuroimage 30(3):787-793

Sereno MI, Huang RS (2006) A human parietal face area contains aligned head-centered visual and tactile maps. Nat Neurosci 9(10):1337-1343

Singer T, Seymour B, O'Doherty J, Kaube H, Dolan RJ, Frith CD (2004) Empathy for pain involves the affective but not sensory components of pain. Science 303(5661):1157-1162

Sripati AP, Yoshioka T, Denchev P, Hsiao SS, Johnson KO (2006) Spatiotemporal receptive fields of peripheral afferents and cortical area $3 \mathrm{~b}$ and 1 neurons in the primate somatosensory system. J Neurosci 26(7):2101-2114

Stringer EA, Chen LM, Friedman RM, Gatenby C, Gore JC (2011) Differentiation of somatosensory cortices by high-resolution fMRI at 7 T. Neuroimage 54(2):1012-1020

Sur M (1980) Receptive fields of neurons in areas $3 b$ and 1 of somatosensory cortex in monkeys. Brain Res 198(2):465-471

Sutherling WW, Levesque MF, Baumgartner C (1992) Cortical sensory representation of the human hand: size of finger regions and nonoverlapping digit somatotopy. Neurology 42(5):1020-1028

Tanosaki M, Suzuki A, Takino R, Kimura T, Iguchi Y, Kurobe Y, Haruta Y, Hoshi Y, Hashimoto I (2002) Neural mechanisms for generation of tactile interference effects on somatosensory evoked magnetic fields in humans. Clinical Neurophysiol Off $\mathbf{J}$ Int Fed Clin Neurophysiol 113(5):672-680

Torquati K, Pizzella V, Della Penna S, Franciotti R, Babiloni C, Romani GL, Rossini PM (2003) "Gating" effects of simultaneous peripheral electrical stimulations on human secondary somatosensory cortex: a whole-head MEG study. Neuroimage 20(3):1704-1713

Unser M, Aldroubi A, Eden M (1993a) B-Spline signal-processing. 1. Theory. IEEE Trans Signal Process 41(2):821-833

Unser M, Aldroubi A, Eden M (1993b) B-Spline signal-processing. 2. Efficient design and applications. IEEE Trans Signal Process 41(2):834-848

Virji-Babul N, Rose A, Moiseeva N, Makan N (2012) Neural correlates of action understanding in infants: influence of motor experience. Brain Behav 2(3):237-242

Wheaton KJ, Thompson JC, Syngeniotis A, Abbott DF, Puce A (2004) Viewing the motion of human body parts activates different regions of premotor, temporal, and parietal cortex. Neuroimage 22(1):277-288

White LE, Andrews TJ, Hulette C, Richards A, Groelle M, Paydarfar J, Purves D (1997) Structure of the human sensorimotor system. I: morphology and cytoarchitecture of the central sulcus. Cereb Cortex 7(1):18-30

Wood R, Gallese V, Cattaneo L (2010) Visuotactile empathy within the primary somatosensory cortex revealed by short-latency afferent inhibition. Neurosci Lett 473(1):28-31

Yoo SS, Freeman DK, McCarthy JJ 3rd, Jolesz FA (2003) Neural substrates of tactile imagery: a functional MRI study. NeuroReport 14(4):581-585

Yousry TA, Schmid UD, Alkadhi H, Schmidt D, Peraud A, Buettner A, Winkler P (1997) Localization of the motor hand area to a knob on the precentral gyrus. A new landmark. Brain $120(\mathrm{Pt}$ 1):141-157

Zarzecki P, Wiggin DM (1982) Convergence of sensory inputs upon projection neurons of somatosensory cortex. Exp Brain Res 48(1):28-42

Zhou YD, Fuster JM (1997) Neuronal activity of somatosensory cortex in a cross-modal (visuo-haptic) memory task. Exp Brain Res 116(3):551-555 